\title{
Analytic approach to the CMB polarization generated by relic gravitational waves
}

\author{
Wen Zhao* and Yang Zhang \\ Astrophysics Center University of Science and Technology of China Hefei, Anhui, China
}

(Received 29 May 2006; published 12 October 2006)

\begin{abstract}
By Polnarev's method we analytically calculate the polarization spectra of the cosmic microwave background radiation (CMB) generated by cosmic relic gravitational waves (RGW). In this analytic approach the physics involved in this generating process is more transparent. Consequently, the effects due to various elements of physics can be isolated easily. In solving the equation for evolution of RGW in the expanding universe, both the sudden transition and the WKB approximation for the scale factor during the radiation-matter transition have been taken. To describe more precisely the decoupling process, we have introduced an analytic expression for the visibility function, consisting of two pieces of half-Gaussian curves. We also include the damping on polarizations due to the photon diffusion up to the second order of the tight coupling. Analytic polarization spectra $C_{l}^{X X}$ have been obtained with the following several improvements over the previous results. 1 . The approximate analytic result is quite close to the numerical one evaluated from the cmbfast code, especially, for the first three peaks of the spectrum that are observable. By using the analytic exact solution of RGW in the sudden transition approximation, we have demonstrated the dependence of $C_{l}^{X X}$ on the dark energy and the baryons. 2. Our analytic half-Gaussian approximation of the visibility function fits better than the usual Gaussian model, and its time integration yields an analytic damping factor, which is parameter-dependent. This improves the spectra by $\sim 30 \%$ around the second and third peaks. 3 . The second order of tight coupling reduces the overall amplitude of $C_{l}^{X X}$ by $58 \%$, comparing with the tight-coupling limit. 4 . The influences of inflation on RGW and on CMB polarization are explicitly demonstrated.
\end{abstract}

DOI: 10.1103/PhysRevD.74.083006

PACS numbers: 98.70.Vc, 04.30.- w, 04.62.+v, 98.80.Es

\section{INTRODUCTION}

Studies on the anisotropies and polarizations of CMB have made great progress, yielding important information of cosmology. Recently, Wilkinson Microwave Anisotropy Probe (WMAP) observational results on the power spectra of the CMB anisotropies and polarizations [1-6] agree well with the prediction of inflation of a spatially flat universe with the nearly scale-invariant and Gaussian spectrum of primordial adiabatic perturbations. Inflationary expansion can generate two types of perturbations $h_{i j}$ of spacetime metric: one is the scalar type (density) of perturbations [7-9], and the other is the tensorial type, i.e., relic gravitational waves (RGW) [7,9-15]. These two kinds of perturbations will enter the Boltzmann equation for the distribution function of photons and influence $\mathrm{CMB}$ during the decoupling. Their impact on the anisotropies and polarizations of CMB are different, especially their respective contributions have not yet completely determined theoretically. Among these two contributions to CMB anisotropies and polarizations, the one from the density perturbations $[16,17]$ is believed to be dominant. However, the tensorial contribution is also important, especially in long wave-length range. Moreover, the magnetic type of polarization of CMB can only be generated by the tensorial perturbations, and it thus provides another channel to

*Electronic address: wzhao7@mail.ustc.edu.cn detect RGW on large scales via CMB detections besides the direct detection by laser interferometers [18-21].

The power spectra of CMB polarizations can be calculated by numerical method [22,23], which gives rather precise predictions. But the semianalytic method is also very helpful in analyzing the underlying physics and in revealing the dependence on the cosmological parameters [24]. A common treatment uses the spherical harmonic functions to expand the Boltzmann equation into a hierarchical set of equations for the multipole moments, then solves each of them step by step separately [22,25-27]. The other treatment was first suggested by Polnarev [14], using a basis of polarization vectors to decompose the Boltzmann equation, ending up with only two equations for the two unknown distribution functions, $\zeta$ and $\beta$, standing for the anisotropy and the polarization, respectively. This treatment is simpler for analytic study and has been further used [28-34]. In this paper, we study the CMB polarizations caused by the RGW in the Polnarev framework. We arrive at a set of analytic formulas for the polarization power spectra, which depends explicitly on the visibility function and on the spectrum of RGW at the decoupling. In our treatment for the ionization history through recombination we introduce a half-Gaussian visibility function, which is more precise than the usual Gaussian fitting. For a more precise description of the evolution of RGW we adopt a WKB approximation for the scalar factor $a(\eta)$ joining the radiation and matter 
dominant eras. When integrating the Boltzmann equation, we find that there are two kinds of damping mechanisms on the CMB anisotropies and polarizations: one is due to the visibility function, causing the so-called "Silk damping" on small scales, and the other is due to the second order modifications in the tight-coupling limit. The latter will result in an overall numerical factor of the spectra for all scales, different from the first one. We also find that the final power spectra depend sensitively on the details of the ratio of the positive and negative modes of RGW at the decoupling time. This complexity prevents one from getting the exact analytic formulas for the power spectra. We introduce a parameter $\alpha$ to describe the combination of the phases from these complex functions.

The organization of this paper is as follows. In Sec. II, as a setup, the framework for description of CMB anisotropies and polarizations is given in Polnarev's method, and the Boltzmann equation for the photon distribution function $f$ is decomposed into a set of two equations of evolution for the functions $\beta$ and $\xi$. In Sec. III, the function $\beta$ and $\xi$ are directly associated with the polarization tensor $P_{a b}$, that contains the Stokes' parameter $Q$ and $U$ as the elements. Employing the tensor spherical harmonic functions $Y_{(l m) a b}^{G}$ and $Y_{(l m) a b}^{C}$ as a basis to expand the tensor $P_{a b}$, the expressions $C_{l}^{G G}$ and $C_{l}^{C C}$ for the electric and magnetic type of polarizations are given in terms of the expansion coefficients. Section IV is devoted to the solution of the equation of RGW, both analytically and numerically. Besides the usual sudden transition with the scale factor $a(\eta)$ of the power-law form for each era, attention is payed to a more smooth transition between the radiation and matter dominant eras, where a WKB approximation on $a(\eta)$ is used. In particular, the spectra $h\left(\eta_{d}\right)$ and $\dot{h}\left(\eta_{d}\right)$ of RGW at the decoupling are given in both approximation schemes. Section V addresses the decoupling process and introduces a two half-Gaussian model for the visibility function $V(\eta)$, which is more precise than the usual Gaussian fitting. In Sec. VI, we integrate analytically the Boltzmann equations up to the second order of the tight coupling, leading to an improvement over the first order calculation. In doing integration, the two pieces of halfGaussian fitting for the visibility function is used, and the complex mixture of the mode functions from the $\dot{h}$ and the Bessel functions is treated with care. We arrive at the analytic expressions for the polarization $\beta$ and for the spectra $C_{l}^{G G}$ and $C_{l}^{C C}$. There appears a damping factor $D(k)$ containing two Silk-like terms with the parameter $\alpha$. In Section VII, we examine the resulting polarization spectra, especially, the damping caused by the visibility function, the height of the spectral amplitude, and the location of peaks. Then we examine various cosmological parameters that influence the spectra, such as the tensorscalar ratio $r$, the baryon $\Omega_{b}$, the dark energy $\Omega_{\Lambda}$ and the primordial spectrum index $n_{T}$. Section VIII is the conclusion that summarizes the main results.

\section{BOLTZMANN EQUATION FOR CMB POLARIZATIONS}

The polarized distribution function of photons is generally represented by a column vector $f=\left(I_{l}, I_{r}, U, V\right)$, and its components are related to the Stokes parameters: $I=$ $I_{l}+I_{r}$ and $Q=I_{l}-I_{r}$. An important property of the Stokes parameters is that, under a rotation $\delta$ about the axis of propagation, the total intensity $I$ and the parameter $V$ are invariant, but $Q$ and $U$ transform as [35]

$$
\left(\begin{array}{c}
Q^{\prime} \\
U^{\prime}
\end{array}\right)=\left(\begin{array}{cc}
\cos 2 \delta & \sin 2 \delta \\
-\sin 2 \delta & \cos 2 \delta
\end{array}\right)\left(\begin{array}{c}
Q \\
U
\end{array}\right)
$$

So $(Q, U)$ together form a spin-2 field according to the coordinate transformation, and can be conveniently described by a $2 \times 2$ polarization tensor $P_{a b}$. For actual detections the photons come from the full sky of 2-sphere with a metric

$$
g_{a b}=\left(\begin{array}{cc}
1 & 0 \\
0 & \sin ^{2} \theta
\end{array}\right)
$$

in the spherical coordinates $(\theta, \phi)$, and the polarization tensor is [31]

$$
P_{a b}(\hat{n})=\frac{1}{2}\left(\begin{array}{cc}
Q(\hat{n}) & -U(\hat{n}) \sin \theta \\
-U(\hat{n}) \sin \theta & -Q(\hat{n}) \sin ^{2} \theta
\end{array}\right),
$$

satisfying $P_{a b}=P_{b a}$, and $g^{a b} P_{a b}=0$, where $\hat{n}$ is a unit vector in the direction $(\theta, \phi)$.

During the era prior to the decoupling in the early universe, the Thomson scattering of anisotropic radiation by free electrons can give rise to the linear polarization only, and does not generate the circular polarization $V$, so we only consider the column vector $f=\left(I_{l}, I_{r}, U\right)$. For the trivial case of a homogeneous and isotropic unpolarized radiation, the distribution is simply $f=f_{0}(\nu)(1,1,0)$, where $f_{0}(\nu)=\frac{1}{e^{h \nu / k T}-1}$ is the usual blackbody distribution function with temperature $T$. The combined effects of the Thomson scattering and the metric perturbations will yield linear polarizations of photons. The time evolution of the photon distribution function is determined by the equation of radiative transfer, essentially the Boltzmann equation [35],

$$
\frac{\partial f}{\partial \eta}+\hat{n}^{i} \frac{\partial f}{\partial x^{i}}=-\frac{d \nu}{d \eta} \frac{\partial f}{\partial \nu}-q(f-J),
$$

where $\hat{n}^{i}$ is the $i$-component of the unit vector in the direction $(\theta, \phi)$ of photon propagation, $q=\sigma_{T} n_{e} a$ is the differential optical depth and has the meaning of scattering rate, $a$ is the scale factor, $\sigma_{T}=6.65 \times 10^{-25} \mathrm{~cm}^{2}$ is the Thomson cross-section, $n_{e}$ is the number density of the free electron, and 


$$
J=\frac{1}{4 \pi} \int_{-1}^{1} d \mu^{\prime} \int_{0}^{2 \pi} d \phi^{\prime} P\left(\mu, \phi, \mu^{\prime}, \phi^{\prime}\right) f\left(\eta, x^{i}, \nu, \mu^{\prime}, \phi^{\prime}\right),
$$

where $\mu=\cos \theta, \mu^{\prime}=\cos \theta^{\prime}$ and

$$
P=\frac{3}{4}\left(\begin{array}{ccc}
\mu^{2} \mu^{\prime 2} \cos 2\left(\phi^{\prime}-\phi\right) & -\mu^{2} \cos 2\left(\phi^{\prime}-\phi\right) & \mu^{2} \mu^{\prime} \sin 2\left(\phi^{\prime}-\phi\right) \\
-\mu^{\prime 2} \cos 2\left(\phi^{\prime}-\phi\right) & \cos 2\left(\phi^{\prime}-\phi\right) & -\mu^{\prime} \sin 2\left(\phi^{\prime}-\phi\right) \\
-2 \mu \mu^{\prime 2} \sin 2\left(\phi^{\prime}-\phi\right) & 2 \mu \sin 2\left(\phi^{\prime}-\phi\right) & 2 \mu \mu^{\prime} \cos 2\left(\phi^{\prime}-\phi\right)
\end{array}\right)
$$

is the phase-matrix. The scattering term $q(f-J)$ in Eq. (3) describes the effect of the Thomson scattering by free electrons, and the term $-\frac{d \nu}{d \eta} \frac{\partial f}{\partial \nu}$ reflects the effect of variation of frequency due to the metric perturbations through the Sachs-Wolfe formula [36]

$$
\frac{1}{\nu} \frac{d \nu}{d \eta}=\frac{1}{2} \frac{\partial h_{i j}}{\partial \eta} \hat{n}^{i} \hat{n}^{j}
$$

In the presence of perturbations $h_{i j}$, either scalar or tensorial, the distribution function will be perturbed and can be generally written as

$$
f(\theta, \phi)=f_{0}\left[\left(\begin{array}{l}
1 \\
1 \\
0
\end{array}\right)+f_{1}(\theta, \phi)\right]
$$

where $f_{1}$ represents the perturbed portion.

The perturbed flat Friedmann-Robertson-Walker (FRW) metric is

$$
d s^{2}=a^{2}(\eta)\left[d \eta^{2}-\left(\delta_{i j}+h_{i j}\right) d x^{i} d x^{j}\right],
$$

where $\eta=\int\left(a_{0} / a\right) d t$ is the conformal time, and $h_{i j}$ are the perturbations with $\left|h_{i j}\right| \ll 1$. In our context, we consider only the tensorial type perturbations $h_{i j}$, representing the RGW. So they are symmetric $h_{i j}=h_{j i}$, traceless $h_{i i}=$ 0 , and transverse $h_{i j, j}=0$. Therefore, there are only two independent modes, corresponding to the + and $\times$ gravitational-wave polarizations.

$$
h_{i j}=h_{i j}^{+}+h_{i j}^{\times}=h^{+} \epsilon_{i j}^{+}+h^{\times} \epsilon_{i j}^{\times} .
$$

Taking the direction of propagation of the GW in the direction of $\hat{z}$, i.e., $\hat{k}=\hat{z}$, then the polarization tensors for the GW satisfy

$$
\epsilon_{i j}^{+} \hat{n}_{i} \hat{n}_{j}=\sin ^{2} \theta \cos 2 \phi, \quad \epsilon_{i j}^{\times} \hat{n}_{i} \hat{n}_{j}=\sin ^{2} \theta \sin 2 \phi .
$$

In cosmological context, it is usually assumed that the two components $h^{+}$and $h^{\times}$have the same magnitude and are of the same statistical properties. To simplify the Boltzmann Eq. (3), for the $h_{i j}=h^{+} \epsilon_{i j}^{+}$polarization, one writes the perturbed distribution function $f_{1}$ in the form [14]

$$
f_{1}=\frac{\zeta}{2}\left(1-\mu^{2}\right) \cos 2 \phi\left(\begin{array}{l}
1 \\
1 \\
0
\end{array}\right)+\frac{\beta}{2}\left(\begin{array}{c}
\left(1+\mu^{2}\right) \cos 2 \phi \\
-\left(1+\mu^{2}\right) \cos 2 \phi \\
4 \mu \sin 2 \phi
\end{array}\right) .
$$

For the $h_{i j}=h^{\times} \epsilon_{i j}^{\times}$polarization, one writes $f_{1}$ in the form

$$
f_{1}=\frac{\zeta}{2}\left(1-\mu^{2}\right) \sin 2 \phi\left(\begin{array}{l}
1 \\
1 \\
0
\end{array}\right)+\frac{\beta}{2}\left(\begin{array}{c}
\left(1+\mu^{2}\right) \sin 2 \phi \\
-\left(1+\mu^{2}\right) \sin 2 \phi \\
-4 \mu \cos 2 \phi
\end{array}\right)
$$

where $\zeta$ represents the anisotropy of photon distribution since $\zeta \propto I_{l}+I_{r}=I$, and $\beta$ represents the polarization of photons since $\beta \propto I_{l}-I_{r}=Q$. Both $\zeta$ and $\beta$ are to be determined by solving the Boltzmann equation. For the $h_{i j}=h^{+} \epsilon_{i j}^{+}$polarization, one substitutes $f$ into Eq. (3). Upon taking Fourier transformation, retaining only the terms linear in the perturbation $h_{i j}$, and performing the integration over $d \mu$, one arrives at a set of two differential equations $[14,33,34]$,

$$
\dot{\xi}_{k}+[i k \mu+q] \xi_{k}=\frac{d \ln f_{0}}{d \ln \nu} \dot{h}_{k}^{+},
$$

$$
\begin{aligned}
\dot{\beta}_{k}+[i k \mu+q] \beta_{k}= & \frac{3 q}{16} \int_{-1}^{1} d \mu^{\prime}\left[\left(1+\mu^{\prime 2}\right)^{2} \beta_{k}\right. \\
& \left.-\frac{1}{2}\left(1-\mu^{\prime 2}\right)^{2} \xi_{k}\right] .
\end{aligned}
$$

For the $h_{i j}=h^{\times} \epsilon_{i j}^{\times}$polarization, the resulting equations are the same as the above with $\dot{h}^{+}$being replaced by $\dot{h}^{\times}$. In Eq. (10) and (11) $\xi_{k} \equiv \zeta_{k}+\beta_{k}$, $k$ is the wavenumber, $\xi_{k}$, $\beta_{k}$, and $h_{k}^{+}$are the Fourier modes of $\xi, \beta$, and $h^{+}$, respectively, and the over dot "." denotes $d / d \eta$. In the following, for simplicity, we will omit the subindex $k$ of the function $\xi_{k}, \beta_{k}$ and $h_{k}$. Moreover, we also drop the GW polarization notation, + or $\times$, since both $h^{+}$and $h^{\times}$are similar in computations. From the structure of Eqs. (10) and (11), one can see that the $\dot{h}$ of GW in Eq. (10) plays the role of a source for the anisotropies $\xi$, which in turn plays the role of a source for the polarization $\beta$ in Eq. (11). Our work is to find the solution of $\beta$, then calculate the CMB polarization power spectra.

As Eq. (10) and (11) contain the differential optical depth $q$, a rather complicated, model-dependent function of time, and the right hand side of Eq. (11) contains an integral over $d \mu^{\prime}$, thus it is difficult to give an exact analytic solution. If $\beta$ and $\xi$ are obtained, one may expand them in terms of the Legendre functions 


$$
\begin{aligned}
& \xi(\eta, \mu)=\sum_{l}(2 l+1) \xi_{l}(\eta) P_{l}(\mu), \\
& \beta(\eta, \mu)=\sum_{l}(2 l+1) \beta_{l}(\eta) P_{l}(\mu),
\end{aligned}
$$

with the Legendre components

$$
\begin{aligned}
& \xi_{l}(\eta)=\frac{1}{2} \int_{-1}^{1} d \mu \xi(\eta, \mu) P_{l}(\mu), \\
& \beta_{l}(\eta)=\frac{1}{2} \int_{-1}^{1} d \mu \beta(\eta, \mu) P_{l}(\mu) .
\end{aligned}
$$

The differential equations Eqs. (10) and (11) for $\xi(\eta, \mu)$ and $\beta(\eta, \mu)$ then become an infinite set of coupled differential equations for $\xi_{l}(\eta)$ and $\beta_{l}(\eta)$.

\section{ELECTRIC AND MAGNETIC TYPES OF CMB POLARIZATIONS}

From Eq. (8) for the definition of $\zeta$ and $\beta$ for the $h^{+} \mathrm{GW}$ polarization, it is seen that the two Stokes parameters $Q$ and $U$ can be constructed as the following $[31,33]$,

$$
\begin{gathered}
Q(\theta, \phi)=\frac{T}{4} \sum_{l}(2 l+1) P_{l}(\cos \theta)\left(1+\cos ^{2} \theta\right) \cos 2 \phi \beta_{l} ; \\
U(\theta, \phi)=\frac{T}{4} \sum_{l}(2 l+1) P_{l}(\cos \theta) 2 \cos \theta \sin 2 \phi \beta_{l} .
\end{gathered}
$$

For scalar functions defined on the 2-sphere, such as the temperature anisotropies $\Delta T$, one commonly uses the spherical harmonic functions $Y_{(l m)}$ as the complete orthonormal basis. But for the $2 \times 2$ tensors defined on the 2sphere, such as $P_{a b}$ in Eq. (2), the following complete orthonormal set of tensor spherical harmonics can be employed [31]:

$$
\begin{gathered}
Y_{(l m) a b}^{\mathrm{G}}=N_{l}\left(Y_{(l m): a b}-\frac{1}{2} g_{a b} Y_{(l m):}{ }_{c}^{c}\right), \\
Y_{(l m) a b}^{\mathrm{C}}=\frac{N_{l}}{2}\left(Y_{(l m): a c} \epsilon_{b}^{c}+Y_{(l m): b c} \epsilon_{a}^{c}\right),
\end{gathered}
$$

where ":" denotes covariant derivative on the 2-sphere, $N_{l} \equiv \sqrt{2(l-2) ! /(l+2) !}$, and

$$
\epsilon_{b}^{a}=\left(\begin{array}{cc}
0 & \sin \theta \\
-1 / \sin \theta & 0
\end{array}\right) \text {. }
$$

They satisfy

$$
\begin{aligned}
\int d \hat{n} Y_{(l m) a b}^{\mathrm{G} *}(\hat{n}) Y_{\left(l^{\prime} m^{\prime}\right)}^{\mathrm{G} a b}(\hat{n}) & =\int d \hat{n} Y_{(l m) a b}^{\mathrm{C} *}(\hat{n}) Y_{\left(l^{\prime} m^{\prime}\right)}^{\mathrm{C} a}(\hat{n}) \\
& =\delta_{l l^{\prime}} \delta_{m m^{\prime}},
\end{aligned}
$$

$$
\int d \hat{n} Y_{(l m) a b}^{\mathrm{G} *}(\hat{n}) Y_{\left(l^{\prime} m^{\prime}\right)}^{\mathrm{C} a b}(\hat{n})=0 .
$$

By construction one sees that $Y_{(l m) a b}^{\mathrm{G}}$ is the gradient (electric type) and $Y_{(l m) a b}^{\mathrm{C}}$ is the curl (magnetic type) of the ordinary spherical harmonics. The polarization tensor can be expanded in this basis as:

$$
\frac{P_{a b}(\hat{n})}{T}=\sum_{l=2}^{\infty} \sum_{m=-l}^{l}\left[a_{(l m)}^{\mathrm{G}} Y_{(l m) a b}^{\mathrm{G}}(\hat{n})+a_{(l m)}^{\mathrm{C}} Y_{(l m) a b}^{\mathrm{C}}(\hat{n})\right]
$$

where expansion coefficients $a_{(l m)}^{\mathrm{G}}$ and $a_{(l m)}^{\mathrm{C}}$ represent the electric and magnetic type components of the polarization, respectively. Note that the sum starts from $l=2$, since RGW generates only perturbations of multipoles from the quadrupoles up. The expansion coefficients are given by

$$
\begin{aligned}
& a_{(l m)}^{\mathrm{G}}=\frac{1}{T} \int d \hat{n} P_{a b}(\hat{n}) Y_{(l m)}^{\mathrm{G} a b *}(\hat{n}), \\
& a_{(l m)}^{\mathrm{C}}=\frac{1}{T} \int d \hat{n} P_{a b}(\hat{n}) Y_{(l m)}^{\mathrm{C} a b *}(\hat{n}),
\end{aligned}
$$

and calculation yields $[31,33]^{1}$ :

$$
\begin{gathered}
a_{l m}^{\mathrm{G}}=\frac{1}{8}\left(\delta_{m, 2}+\delta_{m,-2}\right) \sqrt{2 \pi(2 l+1)}\left[\frac{(l+2)(l+1) \beta_{l-2}}{(2 l-1)(2 l+1)}\right. \\
\left.+\frac{6(l-1)(l+2) \beta_{l}}{(2 l+3)(2 l-1)}+\frac{l(l-1) \beta_{l+2}}{(2 l+3)(2 l+1)}\right] \\
a_{l m}^{\mathrm{C}}=\frac{-i}{4} \sqrt{\frac{2 \pi}{(2 l+1)}}\left(\delta_{m, 2}-\delta_{m,-2}\right) \\
\times\left[(l+2) \beta_{l-1}+(l-1) \beta_{l+1}\right] .
\end{gathered}
$$

where the polarization $\beta$ shows up explicitly in these coefficients. Notice that $\beta_{l}$ in Eqs. (25) and (26) are actually the Fourier mode with the subindex $k$ having been omitted for notational simplicity. Then for each Fourier $k$-mode, the electric type of power spectrum $C_{l}^{\mathrm{GG}}(k)$ is $^{2}$

\footnotetext{
${ }^{1}$ There is a small mistake in the formulas (4.39),(4.40),(4.41) and (4.42) in Ref. [31] and formulas (111),(114) and (115) in Ref. [33], the coefficient of $\frac{6 l(l+1)}{(2 l+3)(2 l-1)}$ should be replaced by $\frac{6(l-1)(l+2)}{(2 l+3)(2 l-1)}$.

${ }^{2}$ In the Ref. [31,33], the bracket of [ ] should be replaced by the absolute value sign $\|$, in case of the values of $\beta$ being imaginary.
} 


$$
\begin{aligned}
C_{l}^{\mathrm{GG}}(k)= & \frac{1}{2 l+1} \sum_{m}\left|a_{l m}^{G}\right|^{2} \\
= & \frac{\pi}{16} \mid \frac{(l+2)(l+1) \beta_{l-2}}{(2 l-1)(2 l+1)}+\frac{6(l-1)(l+2) \beta_{l}}{(2 l+3)(2 l-1)} \\
& +\left.\frac{l(l-1) \beta_{l+2}}{(2 l+3)(2 l+1)}\right|^{2}
\end{aligned}
$$

and similarly for the magnetic type $C_{l}^{\mathrm{CC}}(k)$. These expressions are valid for both $h^{+}$and $h^{\times}$polarizations. Summing over all Fourier modes, and over both + and $\times$ polarization states, one has the following expressions

$$
\begin{aligned}
C_{l}^{\mathrm{GG}}= & \frac{1}{16 \pi} \int \mid \frac{(l+2)(l+1) \beta_{l-2}}{(2 l-1)(2 l+1)}+\frac{6(l-1)(l+2) \beta_{l}}{(2 l+3)(2 l-1)} \\
& +\left.\frac{l(l-1) \beta_{l+2}}{(2 l+3)(2 l+1)}\right|^{2} k^{2} d k, \\
C_{l}^{\mathrm{CC}} & =\frac{1}{4 \pi} \int\left|\frac{(l+2) \beta_{l-1}}{2 l+1}+\frac{(l-1) \beta_{l+1}}{2 l+1}\right|^{2} k^{2} d k .
\end{aligned}
$$

The cross-correlation power spectrum vanishes

$$
C_{l}^{\mathrm{GC}}=\sum_{m=-l}^{m=l} \frac{a_{l m}^{\mathrm{G} *} a_{l m}^{\mathrm{C}}}{2 l+1}=0
$$

since $a_{(l m)}^{\mathrm{G}} \propto\left(\delta_{m, 2}+\delta_{m,-2}\right)$, while $a_{(l m)}^{\mathrm{C}} \propto\left(\delta_{m, 2}-\delta_{m,-2}\right)$. This is expected since the electric type has an even parity and the magnetic type has an odd parity.

\section{EVOLUTION OF GRAVITATIONAL WAVES}

Since RGW term $\dot{h}$ is the source of the CMB polarization in Eq. (10), before one can solve the Boltzmann equation, he needs to know its time evolution, especially around the decoupling time $\eta_{d}$. For both + and $\times$ polarizations, the equation of motion for RGW of mode $k$ is the following:

$$
\ddot{h}+2 \frac{\dot{a}}{a} \dot{h}+k^{2} h=0,
$$

and the initial condition is taken to be

$$
h(\eta=0)=h(k), \quad \dot{h}(\eta=0)=0,
$$

with

$$
\frac{k^{3}}{2 \pi^{2}}|h(k)|^{2}=P_{h}(k)=A_{T}\left(\frac{k}{k_{0}}\right)^{n_{T}}
$$

where $P_{h}(k)$ is the primordial power spectrum of RGW, $A_{T}$ is the amplitude, $k_{0}=0.05 \mathrm{Mpc}^{-1}$ is the pivot wavenumber, and $n_{T}$ is the tensor spectrum index. Inflationary models generically predict $n_{T} \approx 0$, a nearly scale-invariant spectrum. Later we will also see the influence of $n_{T}$ on the CMB polarizations. We have ignored a suppressing effect on RGW by the neutrinos free streaming [37,38], which can slightly reduce the height of the peak at small scales [27].

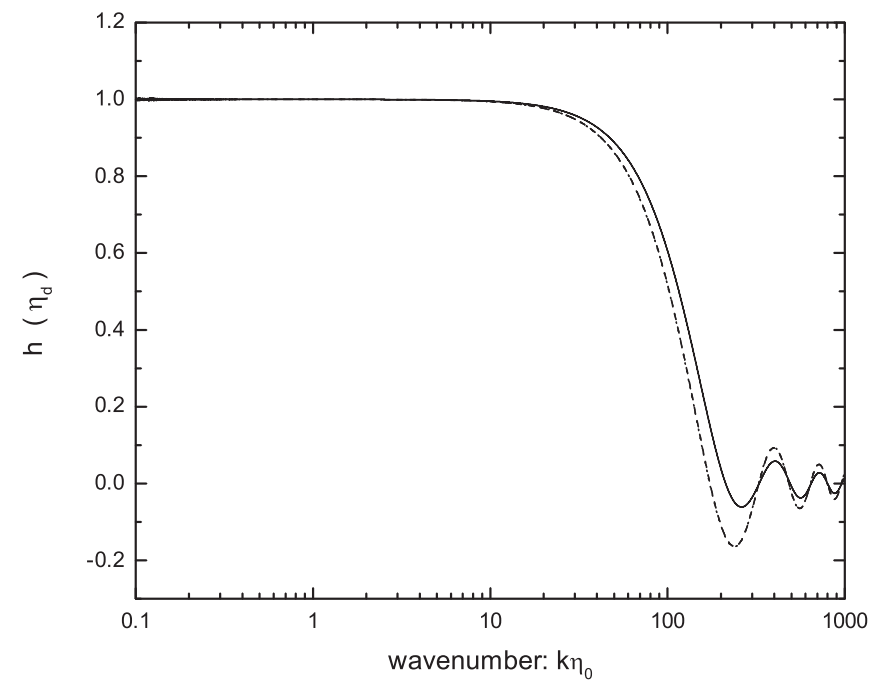

FIG. 1. RGW $h\left(\eta_{d}\right)$ at the decoupling time $\eta_{d}$ depends on the wavenumber $k$. The solid line is the result of the sudden transition approximation, the dash line is of that of the WKB approximation, and the dot line is the numerical result. The latter two lines are nearly overlapped. Here the initial amplitude is rescaled with $h(\eta=0)=h(k)=1$ for demonstrational purpose.

The Eq. (31) depends on the scale factor $a(\eta)$, which is determined by the Friedmann equation

$$
\dot{a}^{2}=H_{0}^{2}\left[\Omega_{r}+a \Omega_{m}+a^{4} \Omega_{\Lambda}\right],
$$

where $H_{0}$ is the present Hubble parameter, $\Omega_{r}, \Omega_{m}$, and $\Omega_{\Lambda}$ are the present fractional densities for the radiation, matter, and dark energy, respectively. Given these fractional densities, say, taking $\Omega_{r}=8.36 \times 10^{-5}, \Omega_{m}=$ $\Omega_{b}+\Omega_{d m}=0.044+0.226, \Omega_{\Lambda}=0.73$, we have solved Eq. (34) numerically. Substituting the resulting $a(\eta)$ and $\dot{a}(\eta)$ into Eq. (31), then the numerical solutions $h(\eta)$ and $\dot{h}(\eta)$ are obtained straightforwardly. The resulting $h\left(\eta_{d}\right)$ and $\dot{h}\left(\eta_{d}\right)$ at the decoupling time $\eta_{d}$ are given as function of $k$ in Fig. 1 and 2, respectively.

Besides the numerical solution, we may use the following analytic expressions of $a(\eta)$, which is a simple approximation for a sudden transiting between two consecutive stages,

$$
a(\eta)= \begin{cases}a_{r} \eta, & \eta \leq \eta_{e} \quad \text { (radiation dominant, } \\ a_{m} \eta^{2}, & \eta_{e}<\eta \leq \eta_{E} \quad \text { (matter dominant, } \\ a_{l} \eta^{-1}, & \eta>\eta_{E} \quad(\Lambda \text { dominant) }\end{cases}
$$

where $a_{r}, a_{m}$, and $a_{l}$ are constants, and can be determined by joining $a(\eta)$ at $\eta_{e}$ and $\eta_{E}$ [39]. For instance, in the $\Lambda$ CDM model ${ }^{3}$ with $\Omega_{b}=0.044, \Omega_{d m}=0.226, \Omega_{\Lambda}=$ 0.73 , taking the redshift $z_{e}=3234$ at the equality of

\footnotetext{
${ }^{3}$ Throughout this paper, we choose the present Hubble parameter $h_{0}=0.72$.
} 


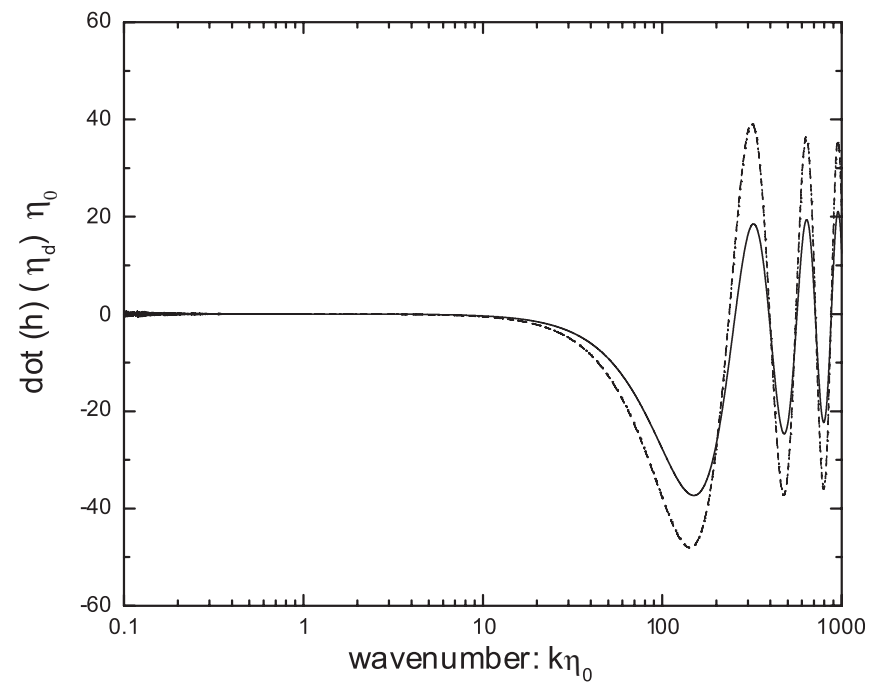

FIG. 2. The time derivative $\dot{h}\left(\eta_{d}\right)$ depends on the wavenumber $k$. The solid line is the result of the sudden transition approximation, the dash line is of that of the WKB approximation, and the dot line is the numerical result. The latter two lines are nearly overlapped. The rescaling $h(k)=1$ is used as in Fig. 1 .

radiation-matter yields the conformal time $\eta_{e} / \eta_{0}=0.007$ (where $\eta_{0}$ is the present conformal time), and taking $z_{E}=$ 0.39 yields $\eta_{E} / \eta_{0}=0.894$. Setting the decoupling time at the redshift $z_{d}=1089$ yields $\eta_{d} / \eta_{0}=0.0195$. Then Eq. (31) has the analytic solution $[26,39,40]$ :

$$
\begin{gathered}
h(\eta)=A_{0} j_{0}(k \eta), \quad\left(\eta \leq \eta_{e}\right), \\
h(\eta)=A_{0}\left(\eta_{e} / \eta\right)\left[A_{1} j_{1}(k \eta)+A_{2} y_{1}(k \eta)\right], \\
\left(\eta_{e}<\eta \leq \eta_{E}\right),
\end{gathered}
$$

with the coefficient

$$
A_{0}=\left[2 \pi^{2} A_{T} k^{-3}\left(\frac{k}{k_{0}}\right)^{n_{T}}\right]^{1 / 2},
$$

determined by the primordial power spectrum, and

$$
\begin{gathered}
A_{1}=\frac{3 k \eta_{e}-k \eta_{e} \cos \left(2 k \eta_{e}\right)+2 \sin \left(2 k \eta_{e}\right)}{2 k^{2} \eta_{e}^{2}}, \\
A_{2}=\frac{2-2 k^{2} \eta_{e}^{2}-2 \cos \left(2 k \eta_{e}\right)-k \eta_{e} \sin \left(2 k \eta_{e}\right)}{2 k^{2} \eta_{e}^{2}} .
\end{gathered}
$$

Note that $h(\eta)$ and $\dot{h}(\eta)$ actually have a subindex $k$, so for each fixed time $\eta h(\eta)$ and $\dot{h}(\eta)$ are functions of the wavenumber $k$. At the decoupling time $\eta_{d}$, the functions $h\left(\eta_{d}\right)$ and $\dot{h}\left(\eta_{d}\right)$ are also plotted against $k$ in Fig. 1 and 2. These figures show that the simple approximation of sudden transition in Eq. (35) is good only in long wavelength region, but in short wavelength $\left(k \eta_{0}>10\right)$ it differs from the numerical one considerably. By the way, using the above analytic expressions of $h(\eta)$, the amplitude of
RGW has been shown to depend explicitly on the dark energy in such a way as $h \propto \Omega_{m} / \Omega_{\Lambda}$ [39].

To improve (35) by a smoother transition between the radiation and the matter dominant eras, one can adopt the WKB approximation to $a(\eta)[27,41]$. Since CMB anisotropies and polarizations are mainly generated around the decoupling, so we are only interested in RGW at time $\eta_{d}$. At this time the dark energy $\Omega_{\Lambda} a^{4}$ is negligibly small and can be omitted. The scale factor can be approximated by

$$
a(\tau)=a_{e} \tau(\tau+2),
$$

where $\tau \equiv(\sqrt{2}-1) \eta / \eta_{e}$, and $a_{e}$ is determined by $a_{0} / a_{e}=1+z_{e}$. When $\tau \ll 2, a(\tau) \rightarrow \tau$, the radiation dominated stage, and when $\tau \gg 2, a \rightarrow \tau^{2}$, the matter dominated stage. The transition between these two stages is smooth. Then the evolution of RGW become

$$
h^{\prime \prime}+2 \frac{a^{\prime}}{a} h^{\prime}+r^{2} h=0,
$$

where $r \equiv k \eta_{e} /(\sqrt{2}-1)$, the prime denotes $d / d \tau$. Although this equation has an analytic solution [27], it is rather lengthy and complex. In this paper, we only employ Eq. (41) as a better approximation of $a(\eta)$ to find the numerical solution of Eq. (42). The resulting $h\left(\eta_{d}\right)$ and $\dot{h}\left(\eta_{d}\right)$ in this WKB approximation are plotted with the dashed lines in Fig. 1 and 2, the results are very good compared with the numerical ones, and the difference between them $\ll 1 \%$. The approximation of (41) is simpler than the numerical $a(\eta)$, and has better precision than the sudden approximation of (35), and will be used to calculate the CMB polarization power spectra.

\section{VISIBILITY FUNCTION FOR DECOUPLING}

Consider the decoupling history of the universe. Before the decoupling, the ionized baryons are tightly coupled to photons by Thomson scattering. Once the temperature falls below a few $\mathrm{eV}$, it becomes favorable for electrons and ions to recombine to form neutral atoms. As the number of charged particles falls, the mean free path of a given photon increases. Eventually, the mean free path becomes comparable to the horizon size and the photon and baryon fluids are essentially decoupled, and the CMB photons last scatter. One can solve the ionization equations during the recombination stage to obtain the visibility function $V(\eta)$, which describes the probability that a given photon last scattered from a particular time $\eta[42,43]$. (Here $V(\eta)$ should not be confused with the Stokes parameter $V$ mentioned at the beginning of Sec. II.) The visibility function depends on the cosmological parameters, especially the baryons $\Omega_{b}$ and the present Hubble parameter $H_{0}$ [24]. In terms of the optical depth $\kappa$, it is given by

$$
V(\eta)=q(\eta) e^{-\kappa\left(\eta_{0}, \eta\right)}
$$

satisfying 


$$
\int_{0}^{\eta_{0}} V(\eta) d \eta=1
$$

where the optical depth function $\kappa\left(\eta_{0}, \eta\right)$ is related to the differential optical depth $q(\eta)$ by $q(\eta)=-d \kappa\left(\eta_{0}, \eta\right) / d \eta$. Figure 3 shows the profile of $V(\eta)$ from the numerical result by the cmbfast, which is sharply peaked around the last scattering. In analytical calculation it is usually fitted by a simple Gaussian function [25,27]

$$
V(\eta)=V\left(\eta_{d}\right) \exp \left(-\frac{\left(\eta-\eta_{d}\right)^{2}}{2 \Delta \eta_{d}^{2}}\right)
$$

where $V\left(\eta_{d}\right)$ is the amplitude at the the decoupling time $\eta_{d}$, and $\Delta \eta_{d}$ is the thickness of decoupling. The analysis of the WMAP data [3] gives the redshift thickness of the decoupling $\Delta z_{d}=195 \pm 2$, which corresponds to $\Delta \eta_{d} / \eta_{0}=0.00143$. Then, taking $V\left(\eta_{d}\right) \eta_{0}=279$ in (45) yields a fitting shown in Fig. 3, which has large error on both sides of $\eta_{d}$, compared with the numerical one. To improve the fitting of $V(\eta)$, we take the following analytic expressions, which consists of two pieces half-Gaussian functions,

$$
\begin{array}{ll}
V(\eta)=V\left(\eta_{d}\right) \exp \left(-\frac{\left(\eta-\eta_{d}\right)^{2}}{2 \Delta \eta_{d 1}^{2}}\right), & \left(\eta \leq \eta_{d}\right) \\
V(\eta)=V\left(\eta_{d}\right) \exp \left(-\frac{\left(\eta-\eta_{d}\right)^{2}}{2 \Delta \eta_{d 2}^{2}}\right), & \left(\eta>\eta_{d}\right)
\end{array}
$$

with $\Delta \eta_{d 1} / \eta_{0}=0.00110, \quad \Delta \eta_{d 2} / \eta_{0}=0.00176$, and $\left(\Delta \eta_{d 1}+\Delta \eta_{d 2}\right) / 2=\Delta \eta_{d}$, satisfying the constraint of

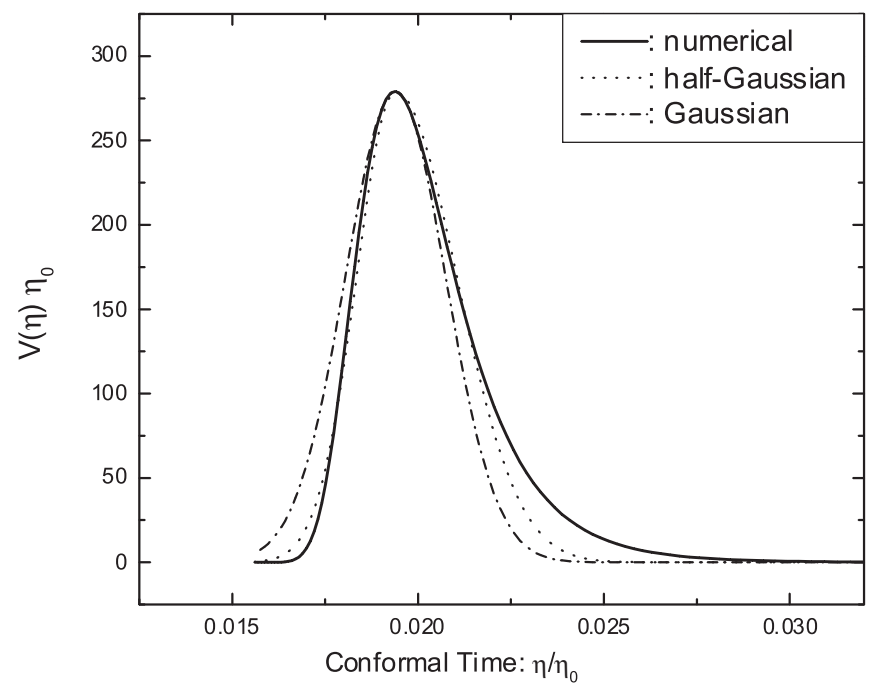

FIG. 3. The curves for the visibility function $V(\eta)$ by the numerical result (solid), by the half-Gaussian fitting (dots) in Eqs. (46) and (47), and by the usual Gaussian fitting (dash) in Eq. (45), respectively. Here $\eta_{d} / \eta_{0}=0.0195$. Comparing with the numerical result, the half-Gaussian fitting improves the Gaussian fitting. Each curve satisfies $\int V d \eta=1$, and the strip area between the Gaussian and the numerical is $\simeq 0.115$.
(44). Figure 3 shows that our half-Gaussian model fits the numerical one much better than the Gaussian fitting. The area enclosed between the curves of the Gaussian and the half-Gaussian is about $\sim 11 \%$ of the total area enclosed under the curve $V(\eta)$. The half-Gaussian fitting improves the description of decoupling, and, nevertheless, is also manageable in analytical computations. As shall be seen later, this different choice of $V(\eta)$ will subsequently cause a variation in the height of the polarization spectra. The expressions (46) and (47) will be used to calculate the approximate analytic polarization power spectra. It turns out that, among the two terms in Eqs. (46) and (47), the result will depend more sensitively on the piece of Eq. (46) with the smaller time interval $\Delta \eta_{d 1}$.

\section{ANALYTIC SOLUTION FOR POLARIZATION}

With the computation of $\dot{h}(\eta)$ and the prescription of $V(\eta)$ above, we are ready to look for the analytic solution of Eqs. (10) and (11). Since the blackbody spectrum $f_{0}(\nu)$ in the Rayleigh-Jeans zone has the property $\frac{d \ln f_{0}(\nu)}{d \ln \nu} \approx 1$, these equations reduce to

$$
\begin{aligned}
\dot{\xi}+[i k \mu+q] \xi=\dot{h}, & \\
\dot{\beta}+[i k \mu+q] \beta= & \frac{3 q}{16} \int_{-1}^{1} d \mu^{\prime}\left[\left(1+\mu^{\prime 2}\right)^{2} \beta\right. \\
& \left.-\frac{1}{2}\left(1-\mu^{\prime 2}\right)^{2} \xi\right] .
\end{aligned}
$$

In Eq. (48) the $\dot{h}$ plays the role of source for the anisotropies $\xi$, while the term $q \xi$ causes $\xi$ to damp. The formal solution of Eq. (48) is

$$
\xi(\eta)=\int_{0}^{\eta} \dot{h}\left(\eta^{\prime}\right) e^{-\kappa\left(\eta, \eta^{\prime}\right)} e^{i k \mu\left(\eta^{\prime}-\eta\right)} d \eta^{\prime} .
$$

In Eq. (49) the integration over $\mu^{\prime}$ contains the integrand functions $\beta$ and $\xi$. Using the expansions for $\xi$ and $\beta$ in Eqs. (12) and (13), and carrying out the $\mu^{\prime}$ integration, then Eq. (49) is

$$
\dot{\beta}+[i k \mu+q] \beta=q G,
$$

where

$$
G(\eta) \equiv \frac{3}{35} \beta_{4}+\frac{5}{7} \beta_{2}+\frac{7}{10} \beta_{0}-\frac{3}{70} \xi_{4}+\frac{1}{7} \xi_{2}-\frac{1}{10} \xi_{0},
$$

where $\xi_{l}$ and $\beta_{l}$ are defined in Eqs. (14) and (15). One might write down a formal solution

$$
\beta(\eta)=\int_{0}^{\eta} G\left(\eta^{\prime}\right) q\left(\eta^{\prime}\right) e^{-\kappa\left(\eta, \eta^{\prime}\right)} e^{i k \mu\left(\eta^{\prime}-\eta\right)} d \eta^{\prime},
$$

and set the time $\eta$ in the above to be the present time $\eta_{0}$,

$$
\beta\left(\eta_{0}\right)=\int_{0}^{\eta_{0}} G\left(\eta^{\prime}\right) V\left(\eta^{\prime}\right) e^{i k \mu\left(\eta^{\prime}-\eta_{0}\right)} d \eta^{\prime},
$$

where $V\left(\eta^{\prime}\right)=q\left(\eta^{\prime}\right) e^{-\kappa\left(\eta_{0}, \eta^{\prime}\right)}$ is the visibility function as 
defined in Eq. (43). However, the difficulty to integrate (53) is that the integrand $G$ contains $\beta_{l}$ and $\xi_{l}$ up to $l=4$, which themselves are not known yet.

One uses the Legendre expansion and write Eqs. (48) and (49) as the following hierarchical set of equations:

$$
\begin{gathered}
\dot{\xi}_{0}=-q \xi_{0}-i k \xi_{1}+\dot{h} \\
\dot{\beta}_{0}=-\frac{3}{10} q \beta_{0}-i k \beta_{1}+q\left(\frac{3}{35} \beta_{4}+\frac{5}{7} \beta_{2}-\frac{3}{70} \xi_{4}\right. \\
\left.+\frac{1}{7} \xi_{2}-\frac{1}{10} \xi_{0}\right) \\
\dot{\xi}_{l}=-q \xi_{l}-\frac{i k}{2 l+1}\left[l \xi_{l-1}+(l+1) \xi_{l+1}\right], \quad \text { for } l \geq 1 \\
\dot{\beta}_{l}=-q \beta_{l}-\frac{i k}{2 l+1}\left[l \beta_{l-1}+(l+1) \beta_{l+1}\right], \quad \text { for } l \geq 1
\end{gathered}
$$

Note that the parameter $1 / q$ has the meaning of the mean free path of photons. In the tight-coupling limit with $q \rightarrow$ $\infty$, the equations reduce to

$$
\dot{\xi}_{0}+q \xi_{0}=\dot{h}
$$

$$
\begin{gathered}
\dot{\beta}_{0}+\frac{3}{10} q \beta_{0}=-\frac{1}{10} q \xi_{0}, \\
\xi_{l}=\beta_{l}=0, \quad l \geq 1 .
\end{gathered}
$$

Then the source function $G(\eta)$ reduces to $G=$ $\left(7 \beta_{0}-\xi_{0}\right) / 10$, and satisfies the equation:

$$
\dot{G}+\frac{3}{10} q G=-\frac{1}{10} \dot{h},
$$

and the formal solution is

$$
G(\eta)=-\frac{1}{10} \int_{0}^{\eta} \dot{h}\left(\eta^{\prime \prime}\right) e^{-(3 / 10) \kappa\left(\eta, \eta^{\prime \prime}\right)} d \eta^{\prime \prime} .
$$

Substitute this expression of $G$ into Eq. (53), yields the formal solution for the polarization in the tight-coupling limit:

$$
\begin{aligned}
\beta\left(\eta_{0}\right) & =\int_{0}^{\eta_{0}} V\left(\eta^{\prime}\right)\left[-\frac{1}{10} \int_{0}^{\eta^{\prime}} \dot{h}\left(\eta^{\prime \prime}\right) e^{-(3 / 10) \kappa\left(\eta^{\prime}, \eta^{\prime \prime}\right)} d \eta^{\prime \prime}\right] e^{i k \mu\left(\eta^{\prime}-\eta_{0}\right)} d \eta^{\prime} \\
& =-\frac{1}{10} \int_{0}^{\eta_{0}} d \eta^{\prime} V\left(\eta^{\prime}\right) e^{i k \mu\left(\eta^{\prime}-\eta_{0}\right)} \int_{0}^{\eta^{\prime}} d \eta^{\prime \prime} \dot{h}\left(\eta^{\prime \prime}\right) e^{-(3 / 10) \kappa\left(\eta^{\prime \prime}\right)+(3 / 10) \kappa\left(\eta^{\prime}\right)},
\end{aligned}
$$

where $\kappa\left(\eta^{\prime}, \eta^{\prime \prime}\right)=\kappa\left(\eta^{\prime \prime}\right)-\kappa\left(\eta^{\prime}\right)$ and $\kappa(\eta) \equiv \kappa\left(\eta_{0}, \eta\right)$ have been used.

However, this result of the tight-coupling limit applies only on scales much larger than the mean free path of photons. On smaller scales the photon diffusion does take place and will cause certain damping in the anisotropies and polarizations. To take care of this effect, we need to expand Eqs. (54) -(57) to the second order of the small coupling parameter $1 / q \ll 1$, and arrive at,

$$
\begin{gathered}
\dot{\xi}_{0}=-q \xi_{0}-i k \xi_{1}+\dot{h}, \\
\dot{\xi}_{1}=-q \xi_{1}-\frac{i k}{3} \xi_{0}, \\
\xi_{l}=0, \quad \text { for } l \geq 2 .
\end{gathered}
$$

Putting $\xi_{0} \propto e^{i \int \omega d \eta}$ and $\xi_{1} \propto e^{i \int \omega d \eta}$ and substituting into Eqs. (64) and (65), ignoring variations of $\omega$ on the expansion scale $\dot{a} / a$, neglecting $\dot{h}$ which is nearly zero at low frequency, shown in Fig. 2, one gets

$$
\omega= \pm \frac{k}{\sqrt{3}}+i q
$$

Thus $\xi_{0}$ will acquire an extra damping factor $e^{-\int q d \eta}$, independent on the wavenumber $k$. This feature is different from the case of the scalar perturbations, where the damping is strong on the small scales [44]. For the polarization $\beta$, we only keep the tight-coupling limit with the equation,

$$
\dot{\beta}_{0}=-\frac{3 q}{10} \beta_{0}-\frac{q}{10} \xi_{0},
$$

and $\beta_{0}$ also gets the factor $e^{-\int q d \eta}$. Thus, taking into account of this effect of the second order of coupling, $G$ in (62) will acquire the extra damping factor $\exp (-\kappa(\eta))$, and, consequently, (63) is modified to

$$
\begin{aligned}
\beta\left(\eta_{0}\right)= & -\frac{1}{10} \int_{0}^{\eta_{0}} d \eta^{\prime} V\left(\eta^{\prime}\right) e^{i k \mu\left(\eta^{\prime}-\eta_{0}\right)} \\
& \times \int_{0}^{\eta^{\prime}} d \eta^{\prime \prime} \dot{h}\left(\eta^{\prime \prime}\right) e^{-(3 / 10) \kappa\left(\eta^{\prime \prime}\right)-(7 / 10) \kappa\left(\eta^{\prime}\right)},
\end{aligned}
$$

where $\exp \left[-\frac{7}{10} \kappa\left(\eta^{\prime}\right)\right]$ has replaced $\exp \left[\frac{3}{10} \kappa\left(\eta^{\prime}\right)\right]$ in Eq. (63). In the above the function $\exp \left(-\frac{7}{10} \kappa\left(\eta^{\prime \prime}\right)\right) \simeq 0$ for $\eta^{\prime \prime}<\eta_{d}$, and $\exp \left(-\frac{7}{10} \kappa\left(\eta^{\prime \prime}\right)\right) \simeq 1$ for $\eta^{\prime \prime}>\eta_{d}$, so it can be approximated as a step function $\exp \left(-\frac{7}{10} \kappa\left(\eta^{\prime \prime}\right)\right) \approx$ $\theta\left(\eta^{\prime \prime}-\eta_{d}\right)$, and moreover, the visibility function $V\left(\eta^{\prime}\right)$ is also peaked about the decoupling $\eta_{d}$. Therefore, as an approximation, one can pull the $\dot{h}\left(\eta^{\prime \prime}\right)$ out of the integration $\int d \eta^{\prime \prime}$, 


$$
\begin{aligned}
\beta\left(\eta_{0}\right)= & -\frac{1}{10} \int_{0}^{\eta_{0}} d \eta V(\eta) e^{i k \mu\left(\eta-\eta_{0}\right)} \dot{h}(\eta) \\
& \times \int_{0}^{\eta} d \eta^{\prime} e^{-(3 / 10) \kappa\left(\eta^{\prime}\right)-(7 / 10) \kappa(\eta)} .
\end{aligned}
$$

Define the integration variable $x \equiv \kappa\left(\eta^{\prime}\right) / \kappa(\eta)$ to replace the variable $\eta^{\prime}$. One can take $d \eta^{\prime}=\frac{d x}{x} \Delta \eta_{d}$ as an approximation, since $V(\eta)$ is peaked around the $\eta_{d}$ with a width $\Delta \eta_{d}$. Then

$$
\begin{aligned}
\beta\left(\eta_{0}\right)= & \frac{1}{10} \Delta \eta_{d} \int_{0}^{\eta_{0}} d \eta V(\eta) e^{i k \mu\left(\eta-\eta_{0}\right)} \dot{h}(\eta) \\
& \times \int_{1}^{\infty} \frac{d x}{x} e^{-(3 / 10) \kappa(\eta) x} e^{-(7 / 10) \kappa(\eta)}
\end{aligned}
$$

Comparing this expression with Eq. (15) and using the expansion formula

$$
e^{i k r \mu}=\sum_{l=0}^{\infty}(2 l+1) i^{l} j_{l}(k r) P_{l}(\mu),
$$

one reads off the expression for the Legendre component of the polarization

$$
\begin{aligned}
\beta_{l}\left(\eta_{0}\right)= & \frac{1}{10} \Delta \eta_{d} i^{l} \int_{0}^{\eta_{0}} d \eta V(\eta) \dot{h}(\eta) j_{l}\left(k\left(\eta-\eta_{0}\right)\right) \\
& \times \int_{1}^{\infty} \frac{d x}{x} e^{-(3 / 10) \kappa(\eta) x} e^{-(7 / 10) \kappa(\eta)} .
\end{aligned}
$$

The integration $\int d \eta$ involves $V(\eta), \dot{h}(\eta)$, and $j_{l}(k(\eta-$ $\left.\eta_{0}\right)$ ). The visibility function $V(\eta)$, either in Eq. (45), or in Eqs. (46) and (47), has generically a factor of the form $e^{-\gamma\left(\eta-\eta_{d}\right)^{2}}$, where $\gamma$ is a constant. As a stochastic quantity, the time-derivative $\dot{h}(\eta)$ contains generally a mixture of oscillating modes, such as $e^{i k \eta}$ and $e^{-i k \eta}$, and so does the spherical Bessel function $j_{l}\left(k\left(\eta-\eta_{0}\right)\right)$. Thus $\dot{h}(\eta) j_{l}\left(k\left(\eta-\eta_{0}\right)\right)$ generally contains terms $\propto e^{-i b k\left(\eta-\eta_{0}\right)}$, where $b \in[-2,2]$. Substituting the half-Gaussian visibility function $V(\eta)$ of Eq. (46) and (47) into the integration $\int d \eta$ of Eq. (70), using the formula

$$
\int_{-\infty}^{\infty} e^{-\gamma y^{2}} e^{i b k y} d y=e^{-\left((b k)^{2} / 4 \gamma\right)} \int_{-\infty}^{\infty} e^{-\gamma y^{2}} d y,
$$

the integration is approximated by

$$
\begin{aligned}
& \int_{0}^{\eta_{0}} d \eta V(\eta) \dot{h}(\eta) j_{l}\left(k\left(\eta-\eta_{0}\right)\right) \\
& \quad \approx D(k) \dot{h}\left(\eta_{d}\right) j_{l}\left(k\left(\eta_{d}-\eta_{0}\right)\right) \int_{0}^{\eta_{0}} d \eta V(\eta) .
\end{aligned}
$$

where

$$
D(k) \equiv \frac{1}{2}\left[e^{-\alpha\left(k \Delta \eta_{d 1}\right)^{2}}+e^{-\alpha\left(k \Delta \eta_{d 2}\right)^{2}}\right] .
$$

If the Gaussian fitting (45) was used, one would have $D(k)=e^{-\alpha\left(k \Delta \eta_{d}\right)^{2}}$. In the above $\alpha$ can take values in $[0,2]$, depending on the mixed modes of the stochastic quantity $\dot{h}(\eta) j_{l}\left(k\left(\eta-\eta_{0}\right)\right)$. Here we will take $\alpha$ as a parameter. Roughly speaking, the function $D(k)$ formally looks like a Fourier transformation of the visibility function and, as a function of $\Delta \eta_{d}$ and $k$, represents the damping caused by photon streaming. This can be interpreted as follows. During the recombination around the last scattering, the visibility function $V(\eta)$ is narrowly centered around the time $\eta_{d}$ with a time width $\Delta \eta_{d}$, thus the last scattering of photons also occur within this time interval statistically. So the smoothing of CMB anisotropies by Thomson scattering is effectively limited within the interval $\Delta \eta_{d}$. Thus a wave of anisotropies $e^{i k \eta}$ will be damped in this interval by a factor $e^{-\left(k \Delta \eta_{d}\right)^{2}}$. The longer the time interval $\Delta \eta_{d}$ is, the more damping the wave suffers. In fact, $\Delta \eta_{d}$ can be also viewed as the thickness of the last scattering surface. Those waves with a wavelength $\lambda$ shorter than the thickness $\Delta \eta_{d}$ will be effectively damped by a factor $e^{-\left(2 \pi \Delta \eta_{d} / \lambda\right)^{2}}$. Thus the shorter the wavelength is, the more damping the wave experiences. It is the factor $D(k)$ that brings down the amplitudes of the spectra on small scales.

The remaining integrations $\int d \eta \int d x$ for $\beta_{l}$ in Eq. (70) are easily carried out

$$
\begin{aligned}
& \int_{0}^{\eta_{0}} d \eta V(\eta) \int_{1}^{\infty} \frac{d x}{x} e^{-(3 / 10) \kappa(\eta) x} e^{(-7 / 10) \kappa(\eta)} \\
& \quad=\int_{0}^{\infty} d \kappa e^{-(17 / 10) \kappa} \int_{1}^{\infty} \frac{d x}{x} e^{-(3 / 10) \kappa x}=\frac{10}{17} \ln \frac{20}{3} .
\end{aligned}
$$

We like to point out that this numerical factor is the outcome from the second order of the tight-coupling, while the first order (the tight-coupling limit) with $G$ being given in (62) would yield a result $\frac{10}{7} \ln \frac{10}{3}$ [27]. Thus, putting all these calculations together, the integration (70) for the polarization is finally given by the following concise expression

$$
\beta_{l}\left(\eta_{0}\right)=\frac{1}{17} \ln \frac{20}{3} i^{l} \Delta \eta_{d} \dot{h}\left(\eta_{d}\right) j_{l}\left(k\left(\eta_{d}-\eta_{0}\right)\right) D(k) .
$$

Substituting this back into Eqs. (28) and (29) yields the final formulas for the polarization spectra

$$
\begin{aligned}
C_{l}^{X X}= & \frac{1}{16 \pi}\left(\frac{1}{17} \ln \frac{20}{3}\right)^{2} \int P_{X l}^{2}\left(k\left(\eta_{d}-\eta_{0}\right)\right) \\
& \times\left|\dot{h}\left(\eta_{d}\right)\right|^{2} \Delta \eta_{d}^{2} D^{2}(k) k^{2} d k,
\end{aligned}
$$

where the superindex " $X$ " labels either " $G$ ", or " $C$ ", the type of of the CMB polarization, for the electric type

$$
\begin{aligned}
P_{G l}(x)= & \frac{(l+2)(l+1)}{(2 l-1)(2 l+1)} j_{l-2}(x)-\frac{6(l-1)(l+2)}{(2 l-1)(2 l+3)} j_{l}(x) \\
& +\frac{l(l-1)}{(2 l+3)(2 l+1)} j_{l+2}(x),
\end{aligned}
$$

and for the magnetic type 


$$
P_{C l}(x)=\frac{2(l+2)}{2 l+1} j_{l-1}(x)-\frac{2(l-1)}{2 l+1} j_{l+1}(x) .
$$

The result (75) is similar to the result in Ref. [27] if we identify $C_{l}^{G G}=C_{E l} / 2$ and $C_{l}^{C C}=C_{B l} / 2$. But here the numerical coefficient is $\frac{1}{17} \ln \frac{20}{3}$, smaller than that in Ref. [27], since we have included the diffusion effect on the source $G$. The reduction of the amplitude is about $\sim 58 \%$ due to the the second order of the tight-coupling. Another difference is the damping factor $D(k)$ having two terms in Eq. (72). Besides, the parameter $\alpha$ is taken to be in the range [0,2], instead of a fixed $\alpha=1 / 2$ as in Ref. [27].

To completely determine $C_{l}^{X X}$, we need to fix the normalization of the initial amplitude $\dot{h}\left(\eta_{d}\right)$ in Eq. (75). What has been observed is the CMB temperature anisotropies, which generally receives contributions from both the scalar and tensor perturbations. The ratio of the contributions

$$
r=\frac{P_{h}\left(k_{0}\right)}{P_{R}\left(k_{0}\right)}
$$

has not been fixed observationally, and only some observational constraints have been given. Based upon the observations of Ly- $\alpha$ forest power spectrum from the SDSS, of the 3-year WMAP, of supernovae, and of galaxy clustering, one can give a constraint of $r<0.22$ at $95 \%$ C.L., or $r<0.37$, at $99.9 \%$ C.L. [45]. We take the ratio $r$ as a parameter in our calculations. WMAP observation [4] indicates that the power spectrum of scalar perturbations has a value

$$
P_{R}\left(k_{0}\right)=2.95 \times 10^{-9} A\left(k_{0}\right),
$$

with the pivot wavenumber $k_{0}=0.05 \mathrm{Mpc}^{-1}$ and the amplitude $A\left(k_{0}\right) \simeq 0.8$. Taking the RGW spectrum index $n_{T}=0$ in Eq. (33) (scale-invariant), then the amplitude of Eq. (33) is given by $A_{T}=2.95 \times 10^{-9} A\left(k_{0}\right) r$, depending on $r$. For instance, if $r=1$ is taken, then $A_{T}=2.36 \times$ $10^{-9}$, and smaller $r$ will yield smaller $A_{T}$ accordingly.

\section{RESULTS AND DISCUSSIONS}

\section{A. Damping effects due to visibility function}

The power spectra of $C_{l}^{G G}$ and $C_{l}^{C C}$, calculated from our analytic formulas (75) and from the numerical cmbfast, have been shown in Fig. 4 and 5, respectively. Both the spectra have the prominent first peak around $l \sim 100$. The approximate analytic result is quite close to the numerical one, especially, for the first three peaks of the spectra that are observable. One sees that $C_{l}^{G G}$ and $C_{l}^{C C}$ at large $l$ sensitively depend on the visibility function $V(\eta)$, that is, on the factor $D(k)$. In particular, for the electric polarization spectrum, our half-Gaussian fitting with $\alpha=1.7$ gives a very good result. The third peak is very close to the numerical one, and the second peak is a little higher. For $\alpha=2$ the second peaks of the spectra are good, but the third peaks are a bit too low. On the other hand, the

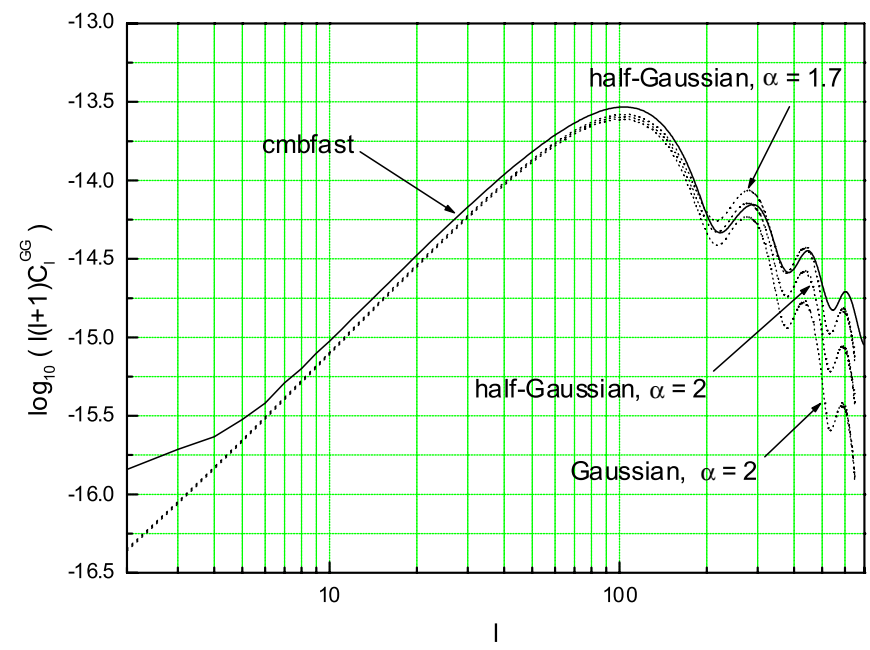

FIG. 4 (color online). The electric polarization spectrum $C_{l}^{G G}$ with the ratio $r=1$. The solid line is the numerical spectrum from the cmbfast code, the upper dot line is from the halfGaussian fitting with $\alpha=1.7$, the middle dot line is from the half-Gaussian fitting with $\alpha=2$ and, and the lower dot line is from the Gaussian fitting with $\alpha=2$. While at large scales these models are close to each other, the half-Gaussian model is better than the Gaussian.

Gaussian fitting with $\alpha=2$ yields a power spectrum too low. This is because $\Delta \eta_{d}>\Delta \eta_{d 1}$, and the Gaussian damping factor $D(k)=e^{-\alpha\left(k \Delta \eta_{d}\right)^{2}}$ reduces the spectrum more than the half-Gaussian factor $D(k)=\frac{1}{2}\left[e^{-\alpha\left(k \Delta \eta_{d 1}\right)^{2}}+\right.$ $\left.e^{-\alpha\left(k \Delta \eta_{d 2}\right)^{2}}\right]$ does. Similar situation is also found for the magnetic polarization in Fig. 5.

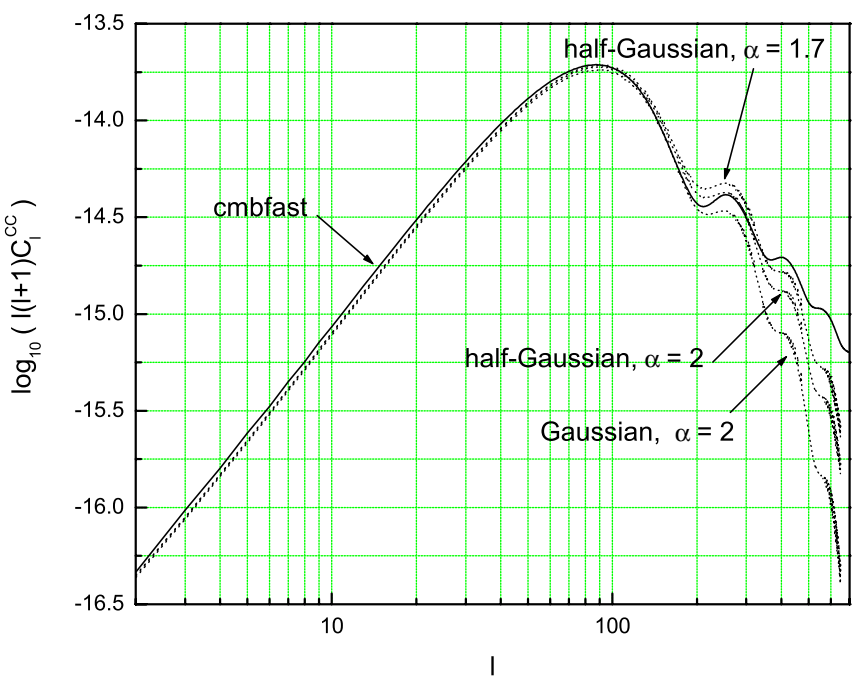

FIG. 5 (color online). The magnetic polarization spectrum $C_{l}^{C C}$ with the ratio $r=1$. The solid line is the numerical spectrum from the cmbfast, the upper dot line is from the half-Gaussian fitting with $\alpha=1.7$, the middle dot line is from the halfGaussian fitting with $\alpha=2$, and the lower one is from the Gaussian fitting with $\alpha=2$. The half-Gaussian model is better than the Gaussian as a fitting formula. 


\section{B. The height of the power spectra}

The analytic expression of Eq. (75) shows that the height of $C_{l}^{X X}$ depends on the amplitude $\left|\dot{h}\left(\eta_{d}\right)\right|$ of RGW at the decoupling time, $C_{l}^{X X} \propto\left|\dot{h}\left(\eta_{d}\right)\right|^{2}$. As has been discussed earlier, for the scale-invariant power spectrum $n_{T} \simeq 0$, $\left|\dot{h}\left(\eta_{d}\right)\right|$ is directly related to the tensor-scalar ratio $r$ in Eq. (78). A larger $r$ yields a larger $\left|\dot{h}\left(\eta_{d}\right)\right|$ and a higher polarization. In Fig. 6 we have plotted the analytic formula (75) for the magnetic type of the polarization spectrum $C_{l}^{C C}$ for three values $r=0.3,0.1,0.01$, respectively, whereby also plotted are the one-sigma sensitivity estimates of the near-term projects, WMAP and Planck satellites [46-48]. The WMAP estimates are based on the measured noise properties of the instrument for an 8-year of operation, and the Planck estimates are based on the noise measurements from the test-bed High Frequency Instrument for a 1.2-year of operation. Figure 6 clearly shows that the spectrum $C_{l}^{C C}$ due to RGW for the inflationary models with $r>0.1$ could be detected by the Planck, but would be difficult for the WMAP.

However, in this discussion of CMB polarizations, we have not considered the effect of cosmic reionization process, which may be directly associated with the the galaxy formation. The reionization will deform the CMB polarization spectra. The recent WMAP result [5] tends to give the optical depth of reionization $\kappa_{r}=0.09 \pm 0.03$. Thus the visibility function $V(\eta)$ will have another peak around a late time $\eta / \eta_{0} \sim 0.27$ besides the cosmic decoupling $\eta_{d}$, and will give an extra contribution to $C_{l}^{C C}$, correspondingly. At present, the reionization process is not well understood yet, and it is difficult to give an analytic formula for this process. Using the numerical cmbfast including the reionization effect, we have plotted $C_{l}^{C C}$ in Fig. 7,

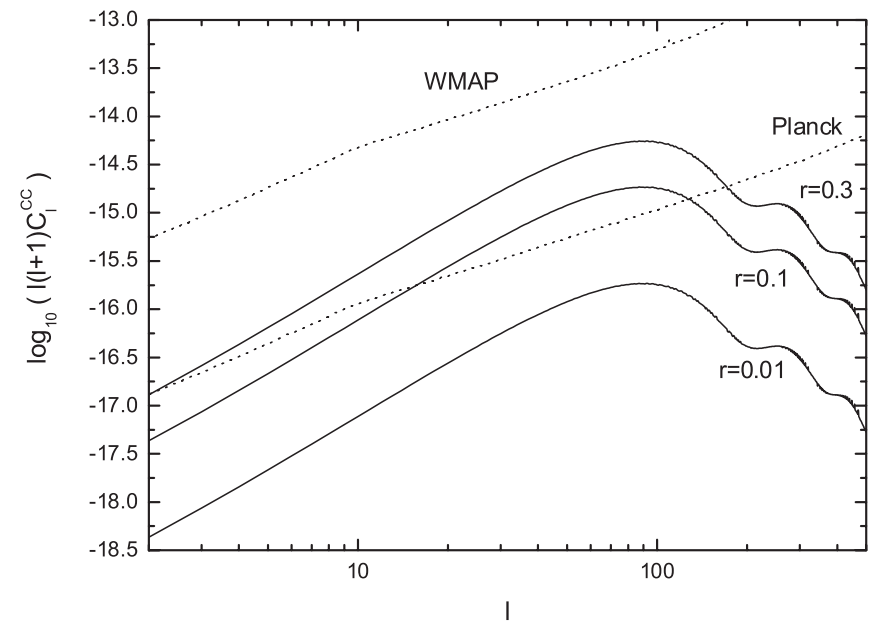

FIG. 6. The WMAP and Planck satellite measurements on the CMB magnetic polarization signal. The three solid curves show the analytic polarization spectra $C_{l}^{C C}$ for the tensor-scalar ratio $r=0.3,0.1,0.01$, respectively, in the $\Lambda \mathrm{CDM}$ universe with $\Omega_{b}=0.044, \Omega_{d m}=0.226, \Omega_{\Lambda}=0.73$.

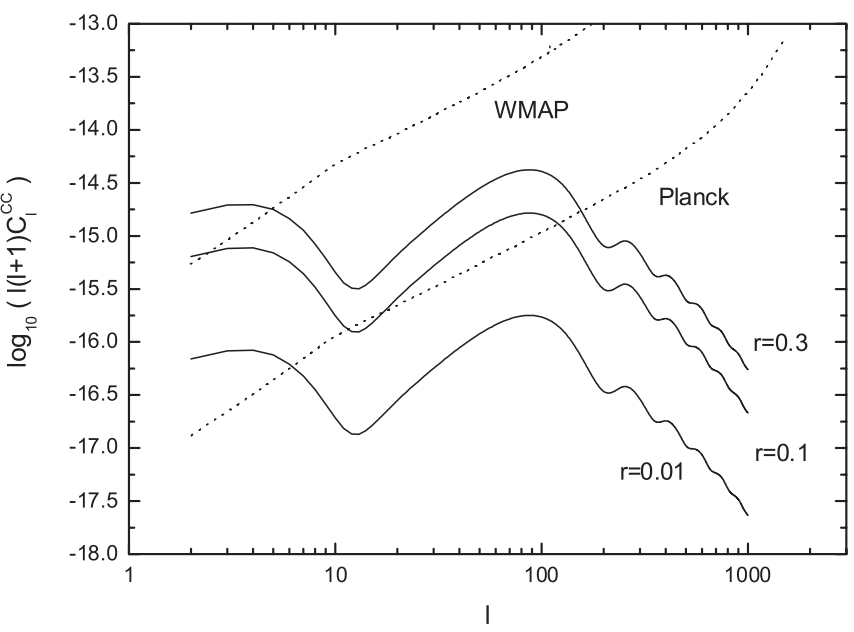

FIG. 7. The WMAP and Planck satellite measurements on the CMB magnetic polarization signal. Now the modifications of the cosmic reionization are included with the reionization optical depth $\kappa_{r}=0.09$. The solid lines show the numerical results using cmbfast. The parameters are the same as in Fig. 6.

where an extra peak of $C_{l}^{C C}$ at $l \sim 6$ is seen. On the observational side, a number of other projects are currently being planned, such as CBI [49], DASI [50], CAPMAP [51], BOOMERANG [52], emphasizing on the detections of the CMB magnetic polarization. The future projects CLOVER [53] and QUIET [54] are expected to detect the magnetic polarization for $r>0.01$, and the project CMBPOL [55] even for $r>10^{-3}$.

\section{The influence of the decoupling width}

Besides the influences by $\mathrm{RGW}$, the spectra $C_{l}^{X X}$ in Eq. (75) also directly depend on the thickness $\Delta \eta_{d}$ of the decoupling and on the damping factor $D(k): C_{l}^{X X} \propto$ $\Delta \eta_{d}^{2} D(k)^{2}$. On very large scales (very small $l$ ), a smaller $\Delta \eta_{d}$ makes the spectra having smaller height. However, on smaller scales (large $l$ ), the effect is complicated since $D(k)$ is also a function of $\Delta \eta_{d}$ and will influence the spectra. The expression $D(k) \equiv \frac{1}{2}\left[e^{-\alpha\left(k \Delta \eta_{d 1}\right)^{2}}+e^{-\alpha\left(k \Delta \eta_{d 2}\right)^{2}}\right]$ in Eq. (72) shows that, for a fixed $k$, the smaller $\Delta \eta_{d}$ leads to a larger $D(k)$. The total effect of $\Delta \eta_{d}$ on the spectra $C_{l}^{X X}$ will be determined by the combination $\propto \Delta \eta_{d}^{2} D(k)^{2}$. The thickness $\Delta \eta_{d}$ is mainly determined by the baryon density $\Omega_{b}$ of the universe. In the flat $\Lambda \mathrm{CDM}$ universe, increasing $\Omega_{b}$ will slightly enhance the decoupling speed, which will make $\Delta \eta_{d}$ becoming smaller [43]. For example, a fitting formula can be used for the optical depth in the $\Lambda \mathrm{CDM}$ universe [24]

$$
\kappa(z)=\Omega_{b}^{c_{1}}\left(\frac{z}{1000}\right)^{c_{2}}, \quad 800<z<1200,
$$

where $c_{1}=0.43$ and $c_{2}=16+1.8 \ln \Omega_{b}$. This fitting function only depends on $\Omega_{b}$. The visibility function $V(\eta)=-\frac{d \kappa}{d \eta} e^{-\kappa}$ is peaked around at $\eta_{d}$. A larger $\Omega_{b}$ 


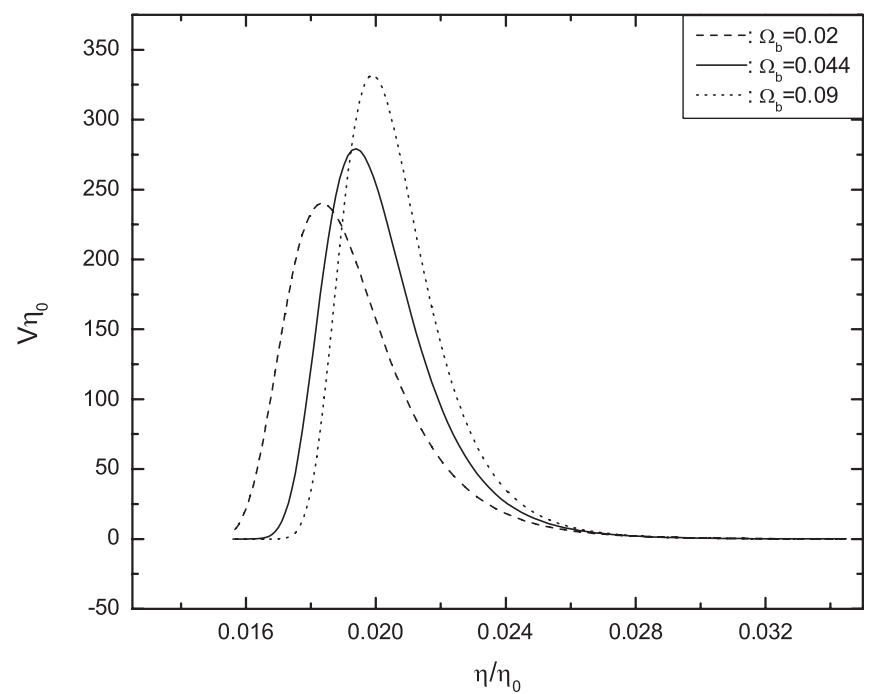

FIG. 8. The dependence of the visibility function $V(\eta)$ on the baryon $\Omega_{b}$ in $\Lambda$ CDM universe with $\Omega_{\Lambda}=0.73$, and $\Omega_{d m}=$ $1-\Omega_{\Lambda}-\Omega_{b}$. The baryon density has been taken to be $\Omega_{b}=$ $0.02,0.044,0.09$, respectively. A larger $\Omega_{b}$ yields a larger $\eta_{d}$ and a smaller $\Delta \eta_{d}$.

corresponds to a narrower $V(\eta)$ and smaller $\Delta \eta_{d}$, as shown in Fig. 8, where three models $\Omega_{b}=0.02,0.044,0.09$ are given. However, the total effect turns out that a higher $\Omega_{b}$ leads to a lower $C_{l}^{C C}$, as shown in Fig. 9 for these three values of $\Omega_{b}$.

\section{The location of the peaks}

From the analytic formula (75), we can also analyze the location of peaks of the power spectra. The factor functions

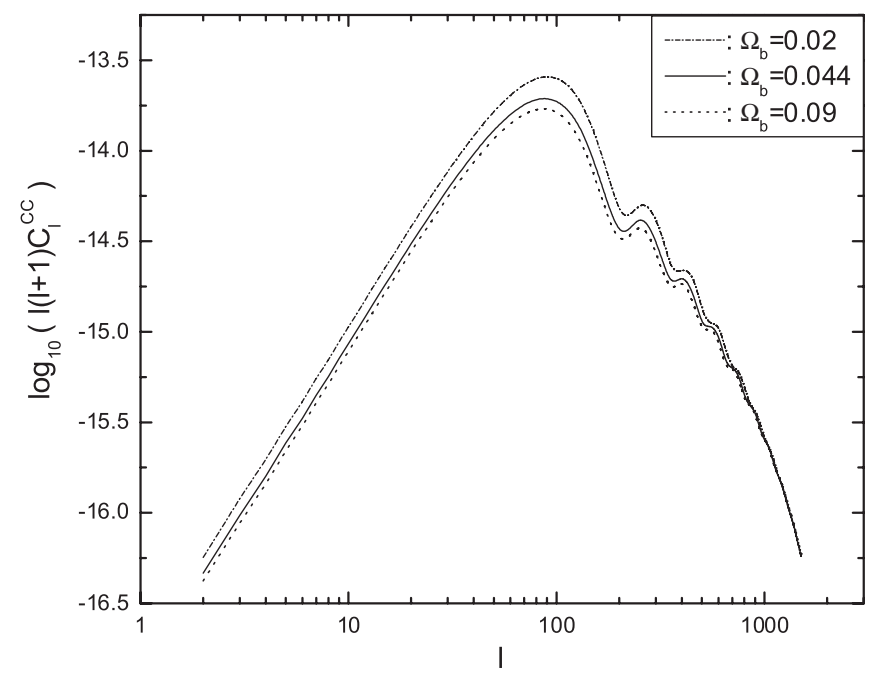

FIG. 9. The magnetic polarization spectrum depends on the baryon $\Omega_{b}$ in $\Lambda$ CDM universe with $\Omega_{\Lambda}=0.73, \Omega_{d m}=1-$ $\Omega_{\Lambda}-\Omega_{b}$, and $r=1$. The baryon $\Omega_{b}=0.02,0.044,0.09$, has been taken, respectively, as in Fig. 8. A larger $\Omega_{b}$ yields a lower amplitude and shifts slightly the peaks to large scales.

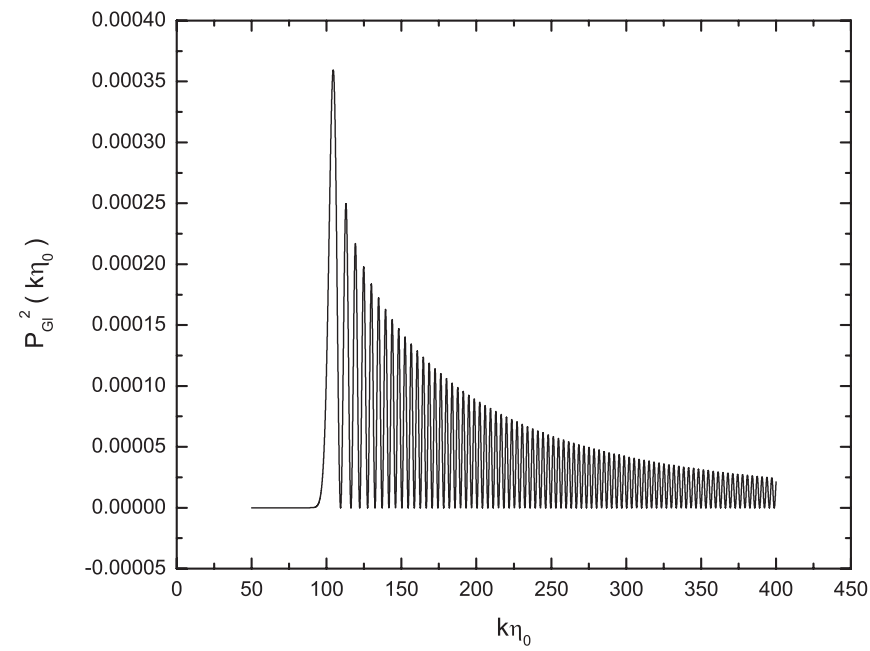

FIG. 10. The factor $P_{G l}^{2}(k)$ as given in Eq. (76) with fixed $l=$ 100 as a function of the wavenumber $k$. Obviously it is peaked around $k \eta_{0} \sim 100$, verifying the relation (82).

$P_{G l}^{2}\left(k\left(\eta_{d}-\eta_{0}\right)\right)$ in Eq. (76) and $P_{C l}^{2}\left(k\left(\eta_{d}-\eta_{0}\right)\right)$ in Eq. (77) are all combination of the spherical Bessel function $j_{l}\left(k\left(\eta_{d}-\eta_{0}\right)\right)$, which is peaked at $l \simeq k\left(\eta_{0}-\eta_{d}\right) \simeq$ $k \eta_{0}$ for $l \gg 1$. In Fig. 10 and $11 P_{G l}$ and $P_{C l}$ are plotted with $l=100$, where it is shown that $P_{G l}$ peaks at $k \eta_{0} \simeq l$, and $P_{C l}$ peaks at $k \eta_{0} \simeq 1.27 l$. So the peak location of the power spectra are directly determined by

$$
\left.C_{l}^{X X} \propto\left|\dot{h}\left(\eta_{d}\right)\right|^{2} k^{2} D^{2}(k)\right|_{k=l / \eta_{0}},
$$

The factor $D(k)$ has a larger damping at larger $l$, so the first peak of the power spectra has the highest amplitude. Let us look at the first peak of $C_{l}^{X X}$, where $D(k) \simeq 1$. Equation (37) gives $\left|\dot{h}\left(\eta_{d}\right)\right|^{2}=A_{0}^{2} k^{2}\left(\eta_{e} / \eta_{d}\right)^{2} \mid A_{1} j_{2}\left(k \eta_{d}\right)+$ $\left.A_{2} y_{2}\left(k \eta_{d}\right)\right|^{2}$. Since $j_{2}\left(k \eta_{d}\right)$ is the increasing mode and the $y_{2}\left(k \eta_{d}\right)$ is the decreasing mode for the waves inside

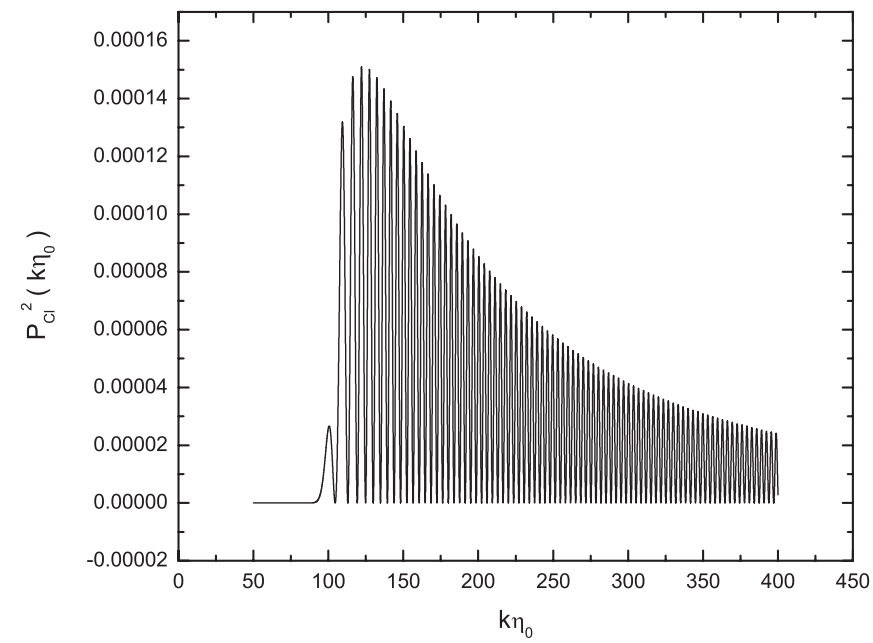

FIG. 11. The factor $P_{C l}^{2}(k)$ as given in Eq. (77) with fixed $l=$ 100 as a function of the wavenumber $k$. It is approximately peaked around $k \eta_{0} \sim 127$, thus Eq. (82) holds as an estimate. 


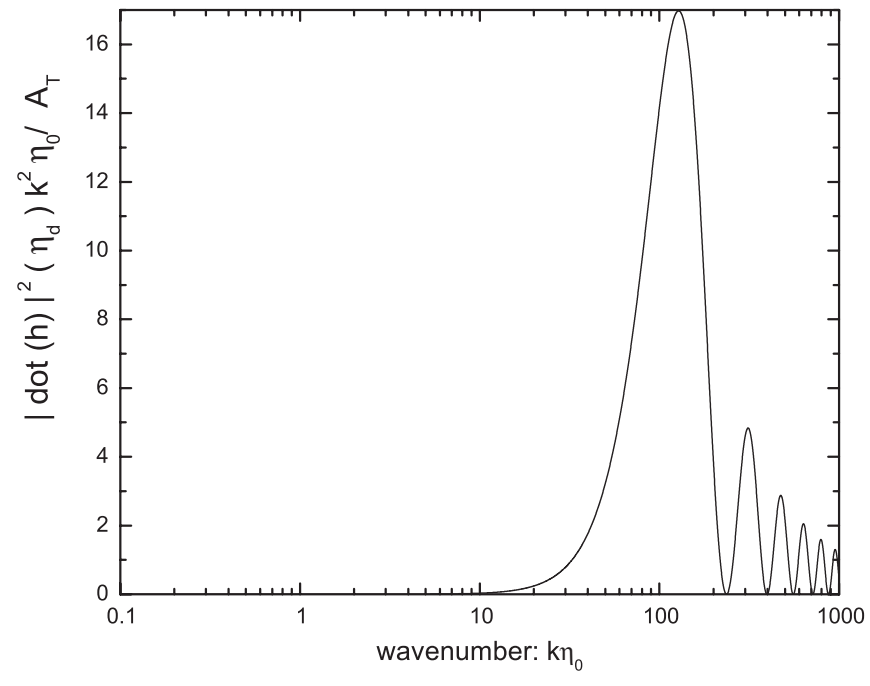

FIG. 12. In the WKB approximation of $a(\eta)$ the factor function $\left|\dot{h}\left(\eta_{d}\right)\right|^{2} k^{2} \eta_{0} / A_{T}$ is peaked around $k \eta_{0} \sim 127$, validating the relation (82) as a fairly good estimate. Here the factor $\left|\dot{h}\left(\eta_{d}\right)\right|^{2} k^{2}$ is multiplied by a factor $\eta_{0} / A_{T}$ for a clear graphical demonstration.

the horizon, so $\left|\dot{h}\left(\eta_{d}\right)\right|^{2} \propto\left|A_{1} j_{2}\left(k \eta_{d}\right)\right|^{2}$. As $j_{2}(x)$ peaks at nearly $x \simeq 3$, so $\left|\dot{h}\left(\eta_{d}\right)\right|^{2}$ peaks at $k \eta_{d} \simeq 3$. Thus $C_{l}^{X X}$ peaks around

$$
l \simeq k \eta_{0} \simeq 3 \eta_{0} / \eta_{d}
$$

For $\Lambda$ CDM universe with $\eta_{d} / \eta_{0}=0.0195$, Eq. (82) gives $k \eta_{0} \sim 154$. This estimate has been based on the analytic solution $h(\eta)$ of the sudden transition of $a(\eta)$, which is an approximation. If we use the WKB approximation, calculation shows that the factor function $\left|\dot{h}\left(\eta_{d}\right)\right|^{2} k^{2}$ in Eq. (81)

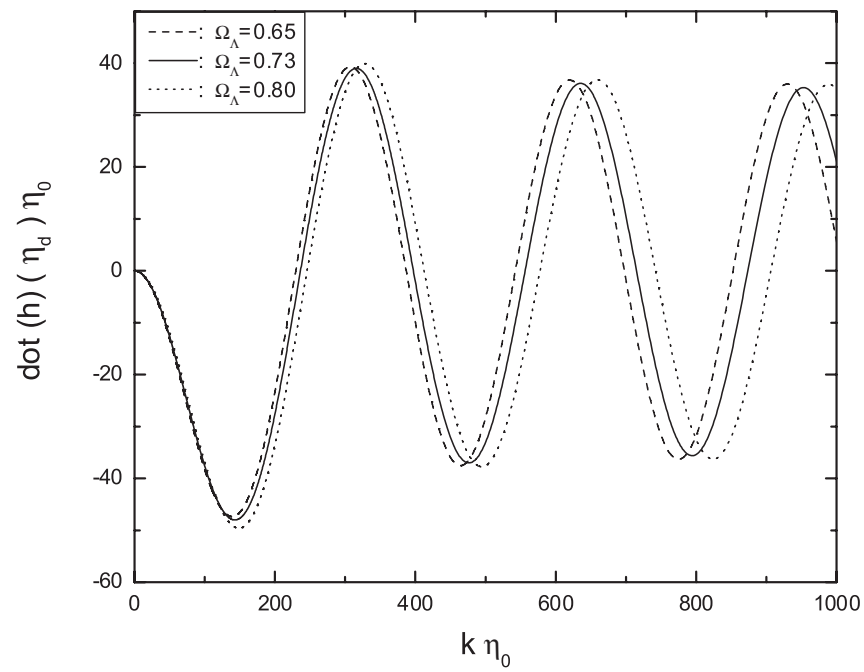

FIG. 13. The dark energy $\Omega_{\Lambda}$ affects the time derivative $\dot{h}\left(\eta_{d}\right)$ at the decoupling. A larger $\Omega_{\Lambda}$ shifts the peaks of $\dot{h}\left(\eta_{d}\right)$ to smaller scales.

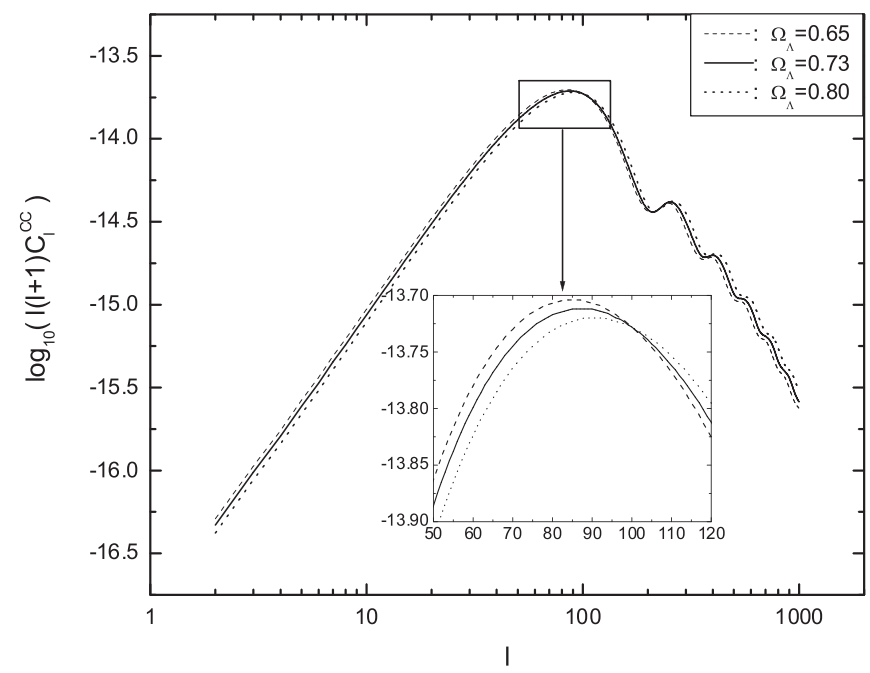

FIG. 14. The magnetic polarization spectrum $C_{l}^{C C}$ depends weakly on $\Omega_{\Lambda}$. A larger $\Omega_{\Lambda}$ yields a lower amplitude and shifts the peaks slightly to smaller scales.

is peaked around $k \eta_{0} \sim 127$, shown in Fig. 12. Therefore, the analytic estimate (82) holds approximately.

The value of $\eta_{0} / \eta_{d}$ is basically determined by the dark energy $\Omega_{\Lambda}$ and the baryon $\Omega_{b}$. For instance, fixing $\Omega_{b}=$ 0.044 and $\Omega_{d m}=1-\Omega_{\Lambda}-\Omega_{b}$, the three models with $\Omega_{\Lambda}=0.65,0.73$, and 0.80 give $\eta_{0} / \eta_{d} \simeq 50.1,51.3$, and 53.6, respectively. Therefore, a larger $\Omega_{\Lambda}$ yields a larger $\eta_{0} / \eta_{d}$, i.e., a longer age of the universe, and $l$ in Eq. (82) is consequently larger. The time derivative of RGW $\dot{h}\left(\eta_{d}\right)$ also depends on $\Omega_{\Lambda}$, as shown in Fig. 13, where it is seen that a larger $\Omega_{\Lambda}$ will shift the peaks of $\dot{h}\left(\eta_{d}\right)$ slightly to smaller scales. Correspondingly, a larger $\Omega_{\Lambda}$ will shift the peaks of $C_{l}^{X X}$ to smaller scales, as demonstrated in Fig. 14. So the conclusion is that a higher dark energy $\Omega_{\Lambda}$ makes the peak of $C_{l}^{X X}$ to locate at larger $l$. This suggests a way to study the cosmic dark energy through CMB polarizations.

The baryon component also influences the decoupling time $\eta_{d}$. A larger $\Omega_{b}$ has a larger decoupling time $\eta_{d}$, and therefore, a smaller $l \simeq 3 \eta_{0} / \eta_{d}$. For fixed $\Omega_{\Lambda}=0.73$ and $\Omega_{d m}=1-\Omega_{\Lambda}-\Omega_{b}$, the three models with $\Omega_{b}=0.02$, 0.044 , and 0.09 , respectively, are given in Fig. 8. The corresponding values are $\eta_{0} / \eta_{d}=54.9,51.3$, and 50.1, respectively. So we have the conclusion that a higher baryon density $\Omega_{b}$ makes the peak to locate at smaller $l$, as is demonstrated in Fig. 9.

\section{E. The influence of the spectrum index $n_{T}$ of RGW on $C_{l}^{X X}$}

The spectrum index $n_{T}$ of RGW in (33) will influence the CMB polarizations $C_{l}^{X X}$. In Fig. 15 three curves of $C_{l}^{C C}$ are plotted for $n_{T}=-0.1,0.0$, and 0.1 , respectively, where the parameters $r=1$ and $\alpha=2$ are taken in the halfGaussian fitting. It is seen that a larger value of $n_{T}$ produces a higher polarization spectrum $C_{l}^{C C}$. The reason for 


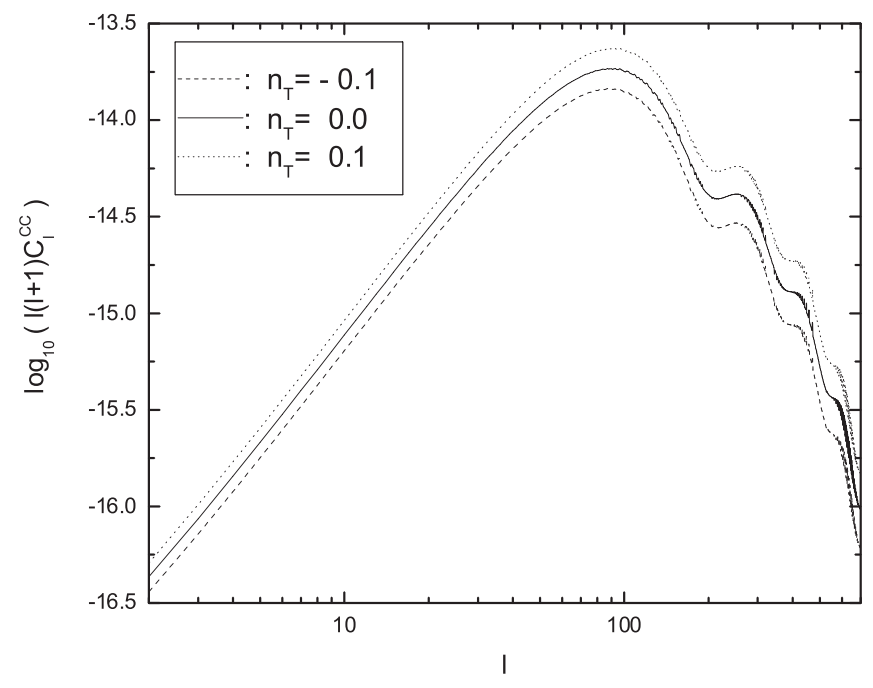

FIG. 15. The magnetic polarization spectrum $C_{l}^{C C}$ depends on the spectrum index $n_{T}$ of RGW. $C_{l}^{C C}$ are plotted for three values of $n_{T}=-0.1,0.0$, and 0.1 , respectively. The parameters are taken: $r=1, \alpha=2$ in the half-Gaussian fitting. A larger $n_{T}$ yields a higher spectrum $C_{l}^{C C}$.

this feature is the following: $C_{l}^{C C} \propto\left|\dot{h}\left(\eta_{d}\right)\right|^{2}$, and $\left|\dot{h}\left(\eta_{d}\right)\right|$ differs from 0 only at larger $k \eta_{0}>50$, as in Fig. 2, and $\left|\dot{h}\left(\eta_{d}\right)\right| \propto k\left|h\left(\eta_{d}\right)\right|$. Since $k^{3}\left|h\left(\eta_{d}\right)\right|^{2} \propto k^{n_{T}}$, so in the range of larger $k$ a larger value $n_{T}$ will give a larger $\left|h\left(\eta_{d}\right)\right|^{2}$ and a larger $C_{l}^{C C}$. The similar behavior also occurs for the electric polarization $C_{l}^{G G}$.

\section{CONCLUSION}

In this paper we present a detailed, and comprehensive study of the CMB polarizations generated by RGW in the Polnarev's method, and have arrived at the analytic formulas of the polarization spectra $C_{l}^{X X}$. As approximate analytic expressions, they are quite close to the numerical result evaluated from the cmbfast code, especially, for the first three peaks of the spectra that are observable. There are several improvements in our approach over the previous results.

(1) The RGW $h(\eta)$ and its derivative $\dot{h}(\eta)$ as the source directly affect the CMB polarization spectra $C_{l}^{X X}$. In our calculations for $h(\eta)$ from its wave equation, both the analytic and numerical treatments have been carried out. In the analytic calculation, we have modeled the scalar factor $a(\eta)$ for various stages of expansion of the universe, including, in particular, the current accelerating one. For the transition between the radiation and the matter dominant eras, besides the sudden transition, we also adopted a smoother description of WKB approximation. While the latter yields the resulting RGW closer to the numerical results, the former has the simpler explicit expressions of $h(\eta)$ and $\dot{h}(\eta)$, which are convenient to employ in analyzing certain physical elements that affect RGW and CMB polarizations. For instances, the location of the peaks of $C_{l}^{X X}$ can be analytically demonstrated with the first peak being located at $l \simeq 3 \eta_{0} / \eta_{d}$, and the dependence on the dark energy $\Omega_{\Lambda}$ and the baryon $\Omega_{b}$, through the decoupling time $\eta_{d}$, is also clearly demonstrated. A lower dark energy $\Omega_{\Lambda}$, and/or a higher baryon density $\Omega_{b}$ tend to shift the peak of $C_{l}^{X X}$ to smaller $l$.

(2) The CMB polarizations depend on the recombination process sensitively. We have given an explicit, and improved expression for the visibility function $V(\eta)$ around the decoupling time, which consists of two pieces of half-Gaussian fitting. This fitting describes the actual decoupling process better than the usual Gaussian one, and is also easy to use for analytic calculations. For example, the damping factor $D(k)$ can be easily written down in the halfGaussian fitting, and, at the same time, it improves the visibility function $V$ by $\sim 11.5 \%$, and the spectra by $\Delta C_{l}^{X X} / C_{l}^{X X} \sim 30 \%$ around the second and the third peaks. Moreover, the damping factor $D(k)$ contains a parameter $\alpha$ in a larger range $[0,2]$, coming from the mixed modes of the stochastic quantity $\dot{h}(\eta) j_{l}\left(k\left(\eta-\eta_{0}\right)\right)$ during the recombination. In particular, the half-Gaussian fitting with $\alpha \in(1.7,2.0)$ gives a reasonably good fitting to the first three peaks of the spectra $C_{l}^{X X}$.

(3) In dealing with the Boltzmann equations analytically, we have worked up to the second order of $1 / q$ of the tight coupling, resulting in an amplitude of $C_{l}^{X X}$ smaller than that in the tight-coupling limit by $58 \%$.

(4) The influences of inflation on the CMB polarizations have been examined, such as the tensor-scalar ratio $r$ and the spectrum index $n_{T}$ of RGW. It is found that a larger value of $n_{T}$ produces higher polarization spectra $C_{l}^{C C}$ and $C_{l}^{G G}$. The possible detections of RGW through CMB polarizations are discussed.

\section{ACKNOWLEDGMENTS}

We acknowledge the using of the cmbfast program [22]. We thank J. R. Pritchard for helpful discussions, the referee for detailed reports. W. Zhao has been partially supported by Graduate Student Research Funding from USTC. Y. Zhang's research work has been supported by the Chinese NSF (10173008), NKBRSF (G19990754), and by SRFDP. 
[1] C.L. Bennett et al., Astrophys. J. Suppl. Ser. 148, 1 (2003).

[2] A. Kogut et al., Astrophys. J. Suppl. Ser. 148, 161 (2003).

[3] D. N. Spergel et al., Astrophys. J. Suppl. Ser. 148, 175 (2003).

[4] H. V. Peiris et al., Astrophys. J. Suppl. Ser. 148, 213 (2003).

[5] D. N. Spergel et al., astro-ph/0603449.

[6] L. Page et al., astro-ph/0603450.

[7] S. Sasaki, Prog. Theor. Phys. 76, 1036 (1986).

[8] E.W. Kolb and M.S. Turner, The Early Universe (Addison-Wesley Publishing Company, Reading, MA, 1990).

[9] V. F. Mukhanov, H. A. Feldman, and R. H. Brandenberger, Phys. Rep. 215, 203 (1992).

[10] V. A. Rubakov, M. Sazhin, and A. Veryaskin, Phys. Lett. B 115, 189 (1982).

[11] R. Fabbri and M.D. Pollock, Phys. Lett. B 125, 445 (1983).

[12] L. Abbott and M. Wise, Nucl. Phys. B237, 226 (1984).

[13] A. Starobinsky, Pis'ma Astron. Zh 11, 323 (1985); [Sov. Astron. Lett. 11, 133 (1985)].

[14] A. Polnarev, Sov. Astron. 29, 6 (1985).

[15] W. Zhao and Y. Zhang, Phys. Rev. D 74, 043503 (2006).

[16] R. Sunyaev and Ya. B. Zeldovich, Astrophys. Space Sci. 7, 3 (1970).

[17] P. J. E. Peebles and J. T. Yu, Astrophys. J. 162, 815 (1970).

[18] B. Abbott et al. (LIGO Scientific Collaboration), Phys. Rev. Lett. 95, 221101 (2005).

[19] http://lisa.nasa.gov/.

[20] http://universe.nasa.gov/program/bbo.html.

[21] N. Seto, S. Kawamura, and T. Nakamura, Phys. Rev. Lett. 87, 221103 (2001).

[22] U. Seljak and M. Zaldarriaga, Astrophys. J. 469, 437 (1996).

[23] A. Lewis, A. Challinor, and A. Lasenby, Astrophys. J. 538, 473 (2000).

[24] W. Hu and N. Sugiyama, Astrophys. J. 444, 489 (1995).

[25] M. Zaldarriaga and D. D. Harari, Phys. Rev. D 52, 3276 (1995).

[26] L. P. Grishchuk, Phys. Rev. D 48, 3513 (1993); Phys. Rev. Lett. 70, 2371 (1993).

[27] J. R. Pritchard and M. Kamionkowski, Ann. Phys. (N.Y.) 318, 2 (2005).

[28] D. Harari and M. Zaldarriaga, Phys. Lett. B 319, 96 (1993).
[29] K. L. Ng and K. W. Ng, Astrophys. J. 445, 521 (1995).

[30] A. Kosowsky, Ann. Phys. (N.Y.) 246, 49 (1996).

[31] M. Kamionkowski, A. Kosowsky, and A. Stebbins, Phys. Rev. D 55, 7368 (1997).

[32] B. Keating et al., Astrophys. J. 495, 580 (1998).

[33] P. Cabella and M. Kamionkowski, astro-ph/0403392.

[34] Y. Zhang, H. Hao, and W. Zhao, Chinese Astronomy and Astrophysics 29, 250 (2005).

[35] S. Chandrasekhar, Rediative Transfer (Dover, New York, 1960).

[36] R. K. Sachs and A.M. Wolfe, Astrophys. J. 147, 73 (1967).

[37] S. Weinberg, Phys. Rev. D 69, 023503 (2004).

[38] D. A. Dicus and W. W. Repko, Phys. Rev. D 72, 088302 (2005).

[39] Y. Zhang, Y.F. Yuan, W. Zhao, and Y.T. Chen, Class. Quant. Grav. 22, 1383 (2005); Y. Zhang, W. Zhao, Y.F. Yuan, and T. Y. Xia, Chin. Phys. Lett. 22, 2538 (2005); Y. Zhang, X.Z. Er, T.Y. Xia, W. Zhao, and H.X. Miao, Class. Quant. Grav. 23, 3783 (2006).

[40] L. P. Grishchuk, Zh. Eksp. Teor. Fiz. 67, 825 (1974); [Sov. Phys. JETP 40, 49 (1974)].

[41] K. W. Ng and A. D. Speliotopoulos, Phys. Rev. D 52, 2112 (1995).

[42] P. J.E. Peebles, Astrophys. J. 153, 1 (1968).

[43] B. J. T. Jones and R. F. G. Wyse, Astron. Astrophys. 149, 144 (1985).

[44] W. Hu and N. Sugiyama, Astrophys. J. 471, 542 (1996).

[45] U. Seljak, A. Slosar, and P. McDonald, astro-ph/0604335.

[46] J. Bock et al., astro-ph/0604101.

[47] http://map.gsfc.nasa.gov/.

[48] Planck Collaboration, astro-ph/0604069.

[49] http://www.astro.caltech.edu/ tjp/CBI/; J. L. Sievers et al., astro-ph/0509203.

[50] http://astro.uchicago.edu/dasi/; E. M. Leitch et al., Astrophys. J. 624, 10 (2005).

[51] http://wwwphy.princeton.edu/cosmology/capmap/; D. Barkats et al., Astrophys. J. 619, L127 (2005).

[52] http://cmb.phys.cwru.edu/boomerang/; G. Polenta et al., Adv. Space Res. 36, 1064 (2005).

[53] Http://www.mrao.cam.ac.uk/ act21/clover.html; A. C. Taylor et al., astro-ph/0407148.

[54] http://quiet.uchicago.edu.

[55] http://universe.nasa.gov/program/inflation.html; L. Verde, H. Peiris, and R. Jimenez, J. Cosmol. Astropart. Phys. 01 (2006) 019. 